\title{
Olanzapine for the prophylaxis and rescue of chemotherapy-induced nausea and vomiting: a systematic review, meta-analysis, cumulative meta-analysis and fragility assessment of the literature
}

\author{
Ronald Chow ${ }^{1,2,3,4}$ (D) Jørn Herrstedt ${ }^{5} \cdot$ Matti Aapro $^{6} \cdot$ Leonard Chiu $^{3,7} \cdot$ Henry Lam $^{3} \cdot$ Elizabeth Prsic $^{2}$. \\ Michael Lock ${ }^{4} \cdot$ Carlo DeAngelis $^{3,8} \cdot$ Rudolph M. Navari $^{9}$
}

Received: 28 October 2020 / Accepted: 3 December 2020 / Published online: 13 January 2021

(C) The Author(s), under exclusive licence to Springer-Verlag GmbH, DE part of Springer Nature 2021

\begin{abstract}
Introduction The aim of this study is to rigorously review the efficacy and safety of olanzapine in defined hematology oncology settings including (1) the setting of highly emetogenic chemotherapy (HEC) and moderately emetogenic chemotherapy (MEC) settings (2) at $5 \mathrm{mg}$ and $10 \mathrm{mg}$ doses, and (3) for response rates for use in the acute, delayed, and overall settings post-MEC and HEC.

Methods Ovid MEDLINE, Embase, and Cochrane Central Register of Controlled Trials were searched through April 23, 2020. The primary efficacy endpoints were the rate of complete response, in the acute ( $0-24 \mathrm{~h}$ post-chemotherapy), delayed ( $24-120 \mathrm{~h}$ post-chemotherapy), and overall (0-120 h post-chemotherapy) phases. The secondary efficacy endpoints were the rates of no nausea and no emesis, for each phase. Safety endpoints were the rate of no serious adverse events (i.e., no grade 3 or 4 toxicities), as assessed by Common Terminology Criteria for Adverse Events (CTCAE) criteria. The Mantel-Haenszel, random-effects analysis model was used to compute risk ratios and accompanying 95\% confidence intervals for each endpoint. For endpoints that statistically favored one arm, absolute risk differences were computed to assess whether there is a $10 \%$ or greater difference, used as the threshold for clinical significance by MASCC/ESMO. Fragility indices were also calculated for each statistically significant endpoint, to quantitatively assess the robustness of the summary estimate. A cumulative meta-analysis was conducted for each efficacy meta-analysis with more than 5 studies, also using the Mantel-Haenszel random-effects analysis model.

Results Three studies reported on olanzapine for the rescue of breakthrough chemotherapy-induced nausea and vomiting (CINV); 22 studies reported on olanzapine in the prophylactic setting. For studies reporting on HEC patients, olanzapinecontaining regimens were statistically and clinically superior in seven of nine efficacy endpoints in the prophylaxis setting. When olanzapine is administered at a $10-\mathrm{mg}$ dose, it is statistically and clinically superior to control patients in eight of nine endpoints among adults. Olanzapine may be effective in the MEC setting and when administered at 5-mg doses, but the paucity of data leads to notable uncertainty.

Conclusion Further RCTs are needed in the setting of MEC patients and administration of olanzapine at a lower 5-mg dose, which may be given to reduce the sedative effect of olanzapine at $10 \mathrm{mg}$.
\end{abstract}

Keywords Olanzapine $\cdot$ Antiemetics $\cdot$ Nausea $\cdot$ Vomiting $\cdot$ Meta-analysis $\cdot$ Systematic review

Ronald Chow

ronald.chow@yale.edu

1 Yale School of Public Health, Yale University, New Haven, CT, USA

2 Yale New Haven Hospital, Yale University, New Haven, CT, USA

3 Sunnybrook Health Sciences Centre, University of Toronto, Toronto, Ontario, Canada

4 London Health Sciences Centre, University of Western Ontario, London, Ontario, Canada
5 Zealand University Hospital, Roskilde, Denmark and University of Copenhagen, Copenhagen, Denmark

6 Genolier Cancer Center, Genolier, Switzerland

7 Columbia University Vagelos College of Physicians and Surgeons, New York, NY, USA

8 Leslie Dan Faculty of Pharmacy, University of Toronto, Toronto, Ontario, Canada

9 Cancer Care Program, Central and South America, World Health Organization, Brimingham, AL, USA 


\section{Introduction}

For cancer patients undergoing chemotherapy, chemotherapyinduced nausea and vomiting (CINV) are two prevalent and potentially treatment-limiting side effects [1]. Female patients and younger patients have been reported to be at greater risk [2-4]. Patients who have experienced vomiting during previous chemotherapy and those with high expectations of severe nausea prior to chemotherapy are at greater risk as well [5].

CINV is classified according to its time of incidence as either acute ( $0-24 \mathrm{~h}$ post-chemotherapy) or delayed (24$120 \mathrm{~h}$ post-chemotherapy). CINV that occurs during the course of chemotherapy despite a prophylactic regimen is termed as breakthrough CINV $[6,7]$.

Only two groups of antiemetics have been developed to target specific biochemical CINV pathways. These include neurokinin $(\mathrm{NK})_{1}$-receptor antagonists (e.g., aprepitant, rolapitant, and netupitant), and serotonergic $(5-\mathrm{HT})_{3}$-receptor antagonists (e.g., ondansetron, palonosetron), whereas dopamine (D) $)_{2}$-receptor antagonists (e.g., prochlorperazine, metoclopramide) initially were developed for different indications [8-11]. Olanzapine was approved by the US Food and Drug Administration as an antipsychotic [12], but has been used off-label as an antiemetic due to its potential to bind to multiple receptors in the CINV pathway, specifically serotonergic 5- $\mathrm{HT}_{2 \mathrm{a}}, 5-\mathrm{HT}_{2 \mathrm{c}}, 5-\mathrm{HT}_{3}, 5-\mathrm{HT}_{6}$, and dopamine $\mathrm{D}_{1}, \mathrm{D}_{2}$, $\mathrm{D}_{3}$, and $\mathrm{D}_{4}$ receptors [13].

Several phase I-II trials first investigated the efficacy and safety of olanzapine [14-19]. A systematic review and metaanalysis of early phase trials reported that $97.2 \%$ and $83.1 \%$ of patients achieved complete response (defined as no emesis and no use of rescue antiemetics) in the acute and delayed phase, respectively [20].

A number of phase III randomized controlled trials were subsequently undertaken and published, and multiple systematic reviews and meta-analyses have been conducted [21-27]. However, no review has separately analyzed antiemetics for highly emetogenic chemotherapy (HEC) and moderately emetogenic chemotherapy (MEC) patients, an important distinction that leads to different clinical guideline recommendations. Notably, the American Society of Clinical Oncology (ASCO) [28] currently recommends olanzapine as part of a four-drug regimen for HEC patients, while the National Comprehensive Cancer Network (NCCN) [29] and the Multinational Association of Supportive Care in Cancer (MASCC)/the European Society for Medical Oncology (ESMO) [30] recommend the four-drug regimen as an option in HEC patients. None of these guidelines, however, recommend olanzapine for MEC patients [28, 30]. Furthermore, each of the published reviews has methodological limitations when appraised using AMSTAR-2, a critical appraisal tool for systematic reviews [31] (Appendix 1 Electronic Supplementary Material).
Given the growing interest in olanzapine and the need for a more rigorous review, the aim of this study is to review the efficacy and safety of olanzapine for the prophylaxis and rescue of CINV through a systematic review and meta-analysis. Furthermore, given the large body of existing data, the aim of this review will be to determine the shortfalls of existing literature to provide future direction for olanzapine research in the CINV setting through a cumulative meta-analysis and fragility assessment.

\section{Methods}

The protocol for this review has been included in Appendix 2 Electronic Supplementary Material. The reporting of this review is conducted in compliance with the Preferred Reporting Items for Systematic Reviews and MetaAnalyses(PRISMA) checklist [32].

\section{Search strategy}

In the interest of conducting a rigorous and comprehensive review, a de novo search strategy was developed to search databases from their beginnings. Ovid MEDLINE, Embase, and the Cochrane Central Register of Controlled Trials were searched from their beginning to April 24, 2020. Search restrictions were placed, so only English-language clinical trials were identified (Appendix 3 Electronic Supplementary Material).

\section{Study selection}

Two independent in-duplicate screenings were conducted. Where disagreements occurred, discussion of discrepancies occurred and consensus achieved, with the input of a senior author if required. Cohen's kappa coefficient was calculated, to report the concordance.

Studies were first screened by title and abstract (level 1 screening). Studies were included after level 1 if they reported on olanzapine in a clinical trial for the setting of CINV. These abstracts then underwent full-text screening (level 2 screening) and were eligible for assessment of quantitative synthesis if they compared an olanzapine-containing regimen in one trial arm to a non-olanzapine-containing regimen in the other trial arm(s). Reference lists of included articles after level 2 screening were also assessed, to identify other potentially relevant randomized controlled trials. Studies with less than 5 patients per arm and non-randomized trials were excluded.

\section{Data extraction}

As with study selection, data extraction was conducted in duplicate and independently. Disagreements were resolved via discussion, to achieve consensus. 
Study demographics of age range, percentage male, chemotherapy emetogenicity, and the difference between the olanzapine regimen and the comparative regimen were noted. The primary efficacy endpoints were the rate of complete response, in the acute ( $0-24 \mathrm{~h}$ post-chemotherapy), delayed (24-120 h post-chemotherapy), and overall ( $0-120 \mathrm{~h}$ postchemotherapy) phases. The secondary efficacy endpoints were the rates of no nausea and no emesis, for each phase. Safety endpoints were the rate of no serious adverse events (i.e., no grade 3 or 4 toxicities, as assessed by Common Terminology Criteria for Adverse Events (CTCAE) criteria), as reported by authors. Grades 1 and 2 toxicities were not extracted for analysis, due to the paucity of data.

When a trial had two olanzapine-containing arms, the data across the two olanzapine arms were summed for analysis and compared to the non-olanzapine-containing arm.

\section{Meta-analysis}

The Mantel-Haenszel, random-effects analysis model was used to compute risk ratios (RRs) and accompanying 95\% confidence intervals for each endpoint. A $p$ value of less than 0.05 was considered statistically significant in the test for overall effect.

Studies were first separately meta-analyzed by regimen intent - whether olanzapine was administered for prophylaxis or for management of breakthrough nausea. It was then analyzed by age, separating studies reporting on adult and children. Adult studies were further meta-analyzed according to chemotherapy emetogenicity, olanzapine dosage, comparative regimens, and study quality; meta-analyses were conducted for the following subgroups of adult studies:

1. HEC studies, as determined by the MASCC/ESMO classification [33]

2. MEC studies, as determined by the MASCC/ESMO classification [33]

3. Olanzapine, administered as $10 \mathrm{mg}$ daily PO

4. Olanzapine, administered as $5 \mathrm{mg}$ daily PO

5. Studies with a double-blind, placebo-controlled design, where the control arm includes a placebo and all antiemetics of the olanzapine-containing arm except for olanzapine itself

6. Studies with an open-controlled design, where the control arm includes all the antiemetics of the olanzapinecontaining arm except for olanzapine itself

7. Studies with an open-controlled design, where the control arm includes antiemetics not included in the olanzapinecontaining arm

For endpoints that statistically favored one arm and had more than 3 included trials, absolute risk differences (RD) were computed to assess whether there is a $10 \%$ or greater difference, deemed to be the threshold for clinical significance by MASCC/ESMO [34]. These analyses were performed using Review Manager (RevMan 5.4) by Cochrane IMS.

\section{Fragility assessment}

Fragility indices were calculated for each statistically significant endpoint by subgroup, to quantitatively assess the robustness of the summary estimate. Determination of the index involves a series of iterative calculations, until the simulated study results change from statistically significant to statistically insignificant according to the Fisher's exact test. Essentially, the index is the number of control patients that would need to change from a nonevent to an event outcome, to change the statistical conclusion of a trial [35]. These analyses were conducted using Stata 16.

\section{Cumulative meta-analysis}

A cumulative meta-analysis was conducted for each efficacy meta-analysis with more than 5 studies, also using the MantelHaenszel random-effects analysis model. These analyses will allow for the assessment of the impact of each trial on the meta-analysis summary effect size and $95 \%$ CI. These analyses were conducted using Comprehensive Meta-Analysis (Version 3) by Biostat.

\section{Assessment of bias}

The Cochrane Risk of Bias tool was used to assess the quality of included randomized controlled trials. Four reviewers (RC, LC, ML, CD) independently assessed bias, after which discussion and consensus was used to resolve any discrepancies. Funnel plots were generated to visually assess for publication bias, for each phase of the three efficacy endpoints where there are 5 or more trials; these were generated using Comprehensive MetaAnalysis (Version 3) by Biostat.

\section{Results}

\section{Included studies}

From the search strategies, 312 records were identified. After removing duplicate records and adding records identified from included trials, 178 records underwent level 1 screening. A total of 34 full-text articles were assessed for eligibility through level 2 screening, at which points 6 were excluded with reason - three were not a randomized controlled trial [36-38], one did not investigate olanzapine in the CINV setting [39], and two did not have an appropriate treatment regimen for inclusion in our review [40, 41]. Of the remaining 28 
articles, 25 randomized controlled trials had extractable data and were included in this systematic review and metaanalysis(Appendix 4 Electronic Supplementary Material). Concordance, as measured by Cohen's Kappa, for level 1 screening was 0.86 , and 0.84 for level 2 (Appendix 5 Electronic Supplementary Material).

Three studies reported on olanzapine for the rescue of breakthrough CINV [42-44]; 22 studies reported on olanzapine in the prophylactic setting [45-66]. Only seven studies (one reporting on rescue of breakthrough CINV, and six reporting on prophylactic CINV) had no corresponding full-text articles $[42,48,53,55,56,60$, 61]. One study reported on olanzapine for children [48]. Among the adult prophylactic studies, 15 reported exclusively on HEC patients [46, 49-52, 55, 56, 58, 60-66], three exclusively on MEC patients [53, 57, 59], and three on a patient population that consists of both HEC and MEC patients [45, 47, 54]. Eight studies compared olanzapine to a double-blind placebo-controlled regimen $[47,52,59,62-66]$ and thirteen used an opened controlled study design - nine studies used a control arm with antiemetics different from the antiemetics in the investigational (olanzapine-containing) arm [46, 49, 51, 53, 55, $56,58,60,61]$ and four used a control arm with the same antiemetics as in the investigational (olanzapinecontaining) arm except for olanzapine [45, 50, 54, 57]. 17 adult prophylactic studies used $10 \mathrm{mg}$ doses of olanzapine $[45,46,49-59,61,62,65,66]$, and 3 studies used $5 \mathrm{mg}[47,60,63] ; 1$ used a mix of $5 \mathrm{mg}$ and $10 \mathrm{mg}$ [64] (Table 1).

\section{Quality of included studies}

The risk of bias assessment for each included study is reported in Appendix 6 Electronic Supplementary Material. Over half of all included studies had high risk of bias, due to concerns around lack of blinding.

\section{Assessment for publication bias of olanzapine for the prophylaxis of CINV}

Funnel plots are presented in Appendix 7, 8 Electronic Supplementary Material. There are no obvious asymmetries, suggesting no obvious concerns of publication bias in this body of literature.

\section{Efficacy of olanzapine for the prophylaxis of CINV in children}

In children, olanzapine was not statistically superior in the acute and overall phases, according to the one study by Long et al.
Efficacy of olanzapine for the prophylaxis of CINV in adults

\section{Complete response}

Acute phase Olanzapine was statistically better than comparative regimens in the acute phase. Among HEC studies, studies using $10 \mathrm{mg}$ olanzapine dosages, studies using a double-blind placebo-controlled design, and open-design studies comparing olanzapine to control regimens of antiemetics not included in the investigational arm, olanzapine was still statistically superior (Fig. 1.1). Olanzapine was clinically superior (risk difference greater than $10 \%$ ) overall, in HEC studies, studies using $10 \mathrm{mg}$ olanzapine doses, and for studies comparing olanzapine in a double-blind placebo-controlled design (Table 2).

Delayed phase Olanzapine was also statistically and clinically superior in the delayed phase. This statistical and clinical superiority prevails in analyses of HEC studies, studies using 10-mg olanzapine doses, studies administering 5-mg of olanzapine, and studies assessing olanzapine in double-blind placebo control studies (Fig. 1.2; Table 2).

Overall phase Olanzapine was statistically and clinically superior in the overall phase among all studies, HEC studies, 10$\mathrm{mg}$ olanzapine studies, 5-mg olanzapine studies, and doubleblind placebo-controlled studies (Fig. 1.3; Table 2).

\section{Nausea control}

For the acute, delayed, and overall phases, olanzapine was statistically superior to comparative regimens. This observation was similarly noted among HEC studies, studies where $10 \mathrm{mg}$ of olanzapine was administered, and double-blind placebo-controlled trials. Olanzapine was also statistically and clinically superior to opendesign studies using a control arm with different antiemetics than used in the investigational (olanzapinecontaining) arm in the delayed and overall phases (Fig. 2; Table 2).

\section{Emesis control}

Neither olanzapine nor control arms were statistically superior to the comparator arm in the acute phase. Olanzapine was both statistically and clinically superior in the delayed and overall phases. Olanzapine was statistically and clinically better in the delayed phase among HEC trials and 10-mg olanzapine trials (Fig. 3; Table 2). 


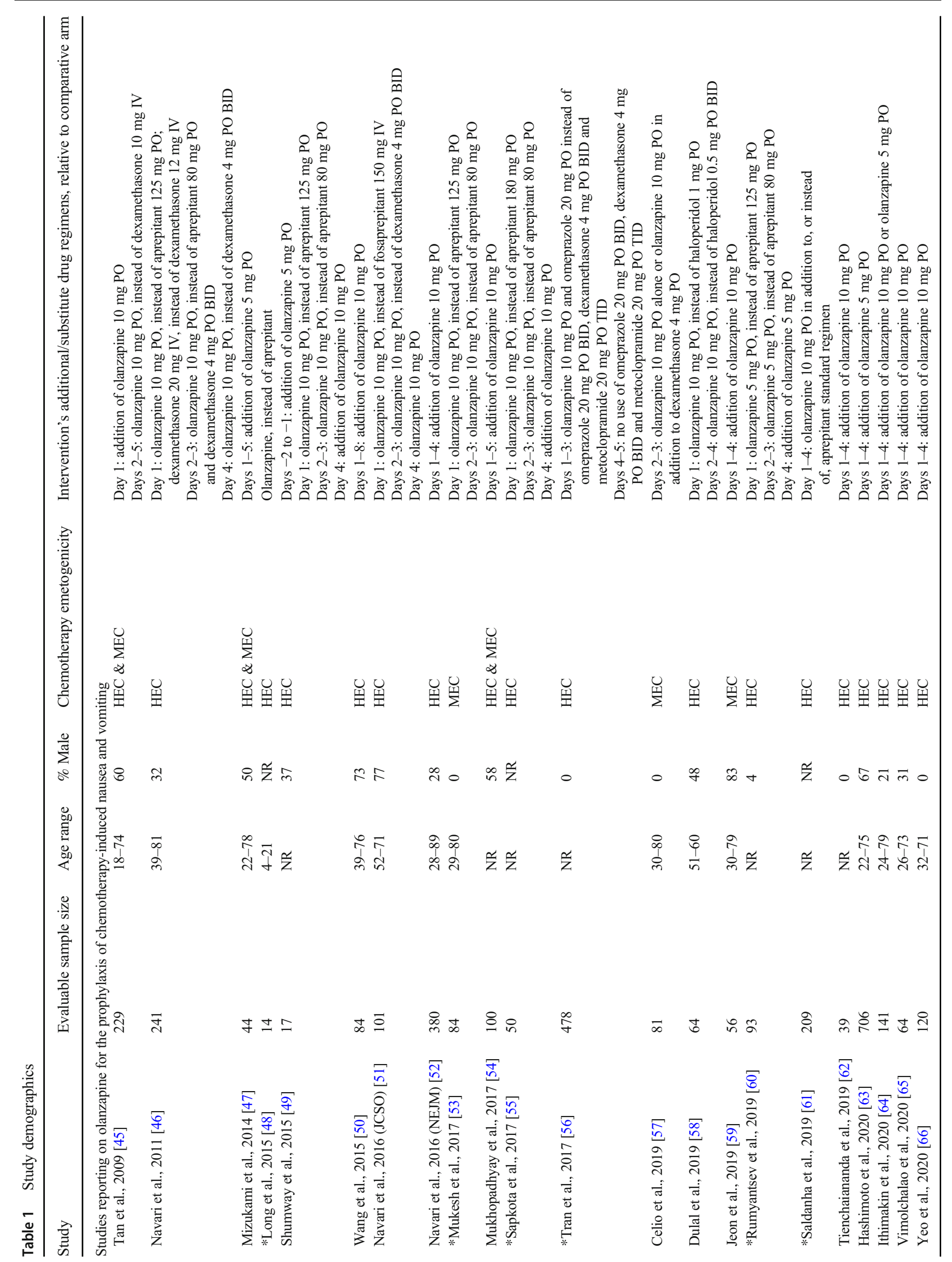




\section{Cumulative meta-analysis and fragility assessment of olanzapine for the prophylaxis of CINV}

Across all three time phases, the meta-analysis results for complete response are the most robust; results reporting on emetic control are the least robust of the three efficacy endpoints (Appendix 9 Electronic Supplementary Material). The most recent trials did not lead to a noticeable effect on the meta-analysis' summary estimate for the endpoints of complete response and nausea control (Appendix 10, 11 Electronic Supplementary Material).

\section{Olanzapine for the rescue of breakthrough CINV}

Olanzapine was statistically superior to comparative regimens with respect to complete control, nausea control, and emetic control, according to the one study reporting on each outcome (Fig. 4). Olanzapine was also clinically superior in all these aforementioned endpoints $-\mathrm{RD}=0.33$ (95\% CI: 0.10-0.56) for complete response in the acute phase, $\mathrm{RD}=0.38$ (95\% CI: 0.18 0.57 ) for complete response in the overall phase, $\mathrm{RD}=0.45$ (95\% CI: $0.28-0.62$ ) for nausea control in the overall phase, and $\mathrm{RD}=0.39$ (95\% CI: $0.21-0.56)$ for emetic control in the overall phase.

\section{Safety of olanzapine for the prophylaxis of CINV}

Olanzapine is as safe as comparative regimens; the risk of serious adverse events is not statistically significant for olanzapine relative to other regimens (Appendix 12, 13 Electronic Supplementary Material).

\section{Discussion}

This review is the most rigorous systematic review to date investigating olanzapine in the CINV setting. A protocol was developed prior to the commencement, risk of bias for studies were assessed, and publication bias was assessed; some or all of these three methodological elements were omitted in prior reviews [21-27].

This review also has the highest statistical power and appraises all the clinically important endpoints. The most recent reviews by Zhou et al. in 2020 included 11 studies with 1107 patients [21]; other reviews by Bahbah et al. in 2019 and Sutherland et al. in 2018 included 9 RCTs with 1572 patients, and 14 trials with 1917 participants, respectively [22, 23]. This

Fig. 1 Efficacy of olanzapine regimens compared to others for the prophylaxis of chemotherapy-induced nausea and vomiting (CINV)Complete response. 1.1 Acute phase. 1.2 Delayed phase. 1.3 Overall phase 
1.1

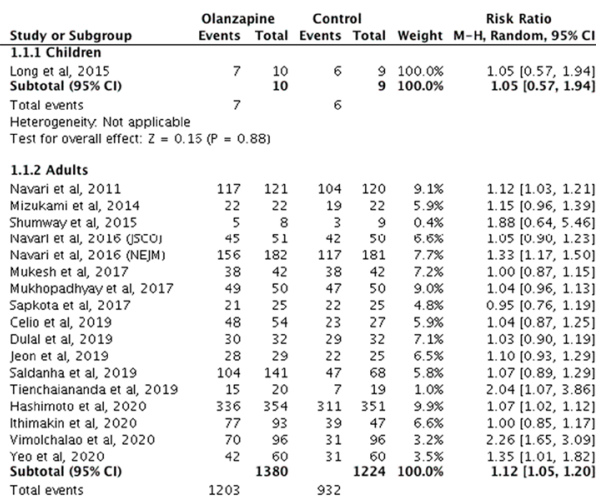

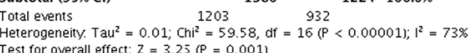

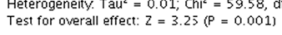

1.1.3 HEC

Navari et a, 2011
Shumway et al, 2015

$\begin{array}{lllll}117 & 121 & 104 & 120 & 10.8 \%\end{array}$

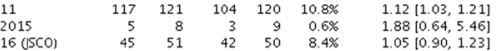

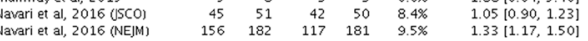

$\begin{array}{llllllll}2017 & 35 & 36 & 34 & 36 & 10.3 \% & 1.33 & 1.117,1.50]\end{array}$

Celio et al, 2019

Lulal et al, 2019

Tienchaiananda et al, 2019

ththimakin et al, 2020

Vimolchalao et al, 2020

Yeo et al, 2020
Subtotal $(95 \% \mathrm{Cl})$

$\begin{array}{lllll}21 & 25 & 22 & 25 & 6.4 \% \\ 48 & 54 & 23 & 27 & 7.5 \%\end{array}$

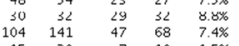

$\begin{array}{lllll}15 & 141 & 7 & 68 & 7.48 \\ 15 & 20 & 79 & 1.58 \\ 3 & 354 & 31 & 351 & 1.58\end{array}$

$\begin{array}{rrrrr} & 354 & 311 & 351 & 11.6 \% \\ 77 & 93 & 39 & 47 & 8.3 \% \\ 70 & 96 & 31 & 96 & 4.4 \% \\ 4 & & 31 & \end{array}$

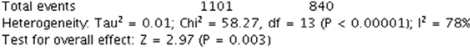

1.1.4 MEC

$\begin{array}{lllllll}\text { Mukesh et al, 2017 } & 38 & 42 & 38 & 42 & 44.1 \% & 1.00[0.87,1.15] \\ \text { Mukhopadhyy et al, 2017 } & 14 & 14 & 13 & 14 & 22.8 \% & 1.07[0.89,1.30]\end{array}$

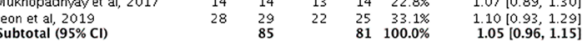
Total events $\quad 80 \quad 73 \quad 76)^{2}=0 \%$

Heterogeneity. Tau ${ }^{2}=0.00 ; \mathrm{Ch}^{2}=0.82$, df $=2(P=0.66) ; \mathbf{l}^{2}=0 \%$

Test for overall effect: $z=1.03(P=0.32)$

1.1.5 HEC/MEC

$\begin{array}{lllllll}\text { 1.1.5 HEC/MEC } & \\ \begin{array}{l}\text { Mizukami et al, 2014 } \\ \text { Subtotal (95\% CI) }\end{array} & 22 & 22 & 19 & 22 & 100.0 \% & 1.15[0.96,1.39] \\ \text { Total events } & 22 & 22 & 19 & 22 & 100.0 \% & 1.15[0.96,1.39]\end{array}$

$1.03[0.93,1.13]$
$0.95[0.76,1.19]$

$1.04[0.87,1.25]$

$1.05[0.90,1.19]$
$1.07[0.89,1.29]$

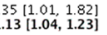

Heterogeneity. Not applicable
Test for overall effect: $Z=1.53(P=0.13)$

1.1.6 Olanzapine $10 \mathrm{mg}$

Navari et al, 2011
Shumwayet al, 2015

Navari et al, 2016 (ISCO)
Navari et a, 2016 (NEIM)

Mukesh et al, 2017

Mukhopadhyyy et al, 2017

Sapkota et al, 2017
Celio et at, 2019

Dulal et al, 2019

leon et al, 2019
Saldanha, et al, 2019

Tienchaiananda et al, 2019

thimakin et al, 2020

Vimolchalao et al, 202

Yeo et al, 2020
Subtotal $(95 \% \mathrm{Cl})$

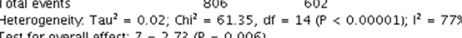

1.1.7 Olanzapine $5 \mathrm{mg}$
Mizukami et al, 2014

et á, 2020

thimakin et al, 2020
Subtotal $(95 \%$ CI)

$\begin{array}{rrrrrr}117 & 121 & 104 & 120 & 9.6 \% & 1.12[1.03,1.21] \\ 5 & 8 & 3 & 9 & 0.7 \% & 1.88[0.64,5.46]\end{array}$

$\begin{array}{rrrrrrr}45 & 51 & 42 & 50 & 7.9 \% & 1.05[0.90,1.231\end{array}$

$\begin{array}{rrrrr}182 & 117 & 181 & 8.7 \% & 1.33[1.17,1.50] \\ 42 & 38 & 42 & 8.3 \% & 1.00[0.87,1.15]\end{array}$

$1.04[0.96,1.13]$

$1.04[0.96,1.13]$
$0.95[0.76,1.19]$

$1.04[0.87,1.25]$

$1.03[0.90,1.19]$

$1.10[0.93,1.29]$
$1.07[0.89,1.29]$

$2.04[1.07,3.86]$

$2.00[0.83,1.20]$

$1.35[1.01,1.82]$
$1.13(1.04,1.24]$

Heterogeneity. $\mathrm{Tau}^{2}=0.00 ; \mathrm{Chi}^{2}=1.19 \%$ dif $=2\langle\mathbf{P}=0.55) ; \mathrm{l}^{2}=0 \%$

1.1.8 Double-Blind Placebo-Controlled Design

Mizukami et al, 2014

Navari et al, 2016 (NE)M)

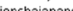

aiananda et al, 2019
noto et al, 2020

thimakin et al, 2020

Vimolchalao et al, 2020

Yeo et al, 2020
Subtotal $95 \% \mathrm{CI})$

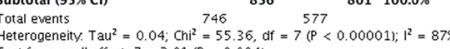

$1.15[0.96,1.39]$
$1.07[1.02,1.12]$
$1.00[0.83,1.20]$

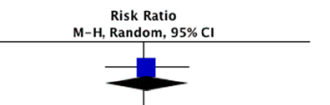

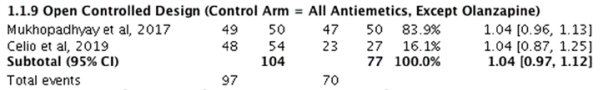

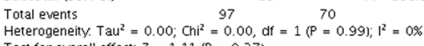

Test for overall effect: $Z=1.11(P=0.27)$

1.1.10 Open Controlled Design (Control Arm = Antiemetics Not In Olanzapine Arm)

Navari et al, 2011
Shumway et al, 2015

Navari et al, 2016 (SCCO

Mukesh et al, 2017

Dula et al, 2019

Saldanha et al, 2019
Subtotal $(95 \% \mathrm{Cl})$

Total events 360

$\begin{array}{rrrrr}\text { (Control Arm = Antiemetics Not In } \\ 117 & 121 & 104 & 120 & 46.6 \% \\ 5 & 8 & 3 & 9 & 0.2 \% \\ 45 & 51 & 42 & 50 & 11.3 \% \\ 38 & 42 & 38 & 42 & 14.5 \% \\ 21 & 25 & 22 & 25 & 5.6 \% \\ 30 & 32 & 29 & 32 & 13.7 \% \\ 104 & 141 & 47 & 68 & 8.0 \% \\ & 420 & & 336 & 100 \%\end{array}$

360
$=4.35, \mathrm{dr}$

$285346 \quad 100.0 \%$

$1.12[1.03,1.21]$
$1.88[0.64,5.46]$
$1.05[0.90,1.23]$
$1.00[0.87,1.15]$
$0.95[0.76,1.19]$
$1.03[0.90,1.19]$
$1.07[0.89,1.29]$
$1.07[1.01,1.13]$

$1.15[0.96,1.39]$
$1.33[1.17,1.50]$

$1.10[0.93,1.29]$

$2.04[1.07,3.86]$
$1.07[1.02,1.12]$

$1.00[0.85,1.17]$
$2.26[1.65,3.09]$

$1.35[1.01,1.82]$
$1.26[1.08,1.48]$

Test for overall effect: $z=2.42(P=0.02)$.

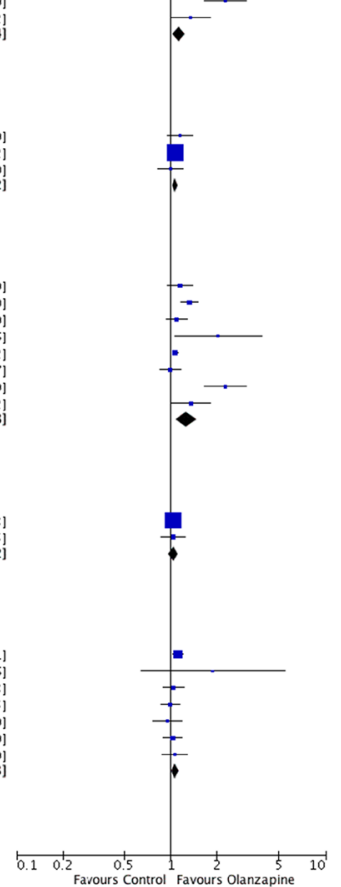


1.2

\begin{tabular}{|c|c|c|c|c|}
\hline bgro & $\begin{array}{l}\text { Olanzapi } \\
\text { Events }\end{array}$ & $\begin{array}{l}\text { pine } \\
\text { Total }\end{array}$ & $\begin{array}{l}\text { Control } \\
\text { Events T }\end{array}$ & \\
\hline & & & & \\
\hline an & & 22 & 16 & \\
\hline $\begin{array}{l}\text { humwayet al, } \\
\text { avari et at, } 20\end{array}$ & & 9 & $\begin{array}{r}5 \\
37\end{array}$ & $\begin{array}{r}99 \\
50\end{array}$ \\
\hline 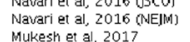 & $\begin{array}{r}39 \\
109\end{array}$ & 163 & 88 & $\begin{array}{l}30 \\
168\end{array}$ \\
\hline al, 2017 & $\begin{array}{l}31 \\
48\end{array}$ & $\begin{array}{l}42 \\
50\end{array}$ & $\begin{array}{l}35 \\
21\end{array}$ & $\begin{array}{l}42 \\
50\end{array}$ \\
\hline kota et al 2017 & 19 & 25 & 18 & 25 \\
\hline 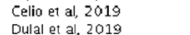 & & 54 & 22 & 27 \\
\hline & & & & 32 \\
\hline & & 141 & 48 & \\
\hline S & 280 & 354 & 2313 & 351 \\
\hline 020 & $\begin{array}{l}65 \\
65\end{array}$ & $\begin{array}{l}93 \\
96\end{array}$ & 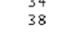 & \\
\hline & & & & \\
\hline
\end{tabular}

Risk Ratio
Risk Ratio
M-H, Random, $95 \% \mathrm{Cl}$

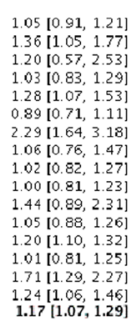

(t)otal

1033

$\begin{array}{rrr}45 & 8.2 \% \\ 1192 & 100.0 \%\end{array}$

Heterogeneity. $\operatorname{Tau}^{2}=0.02 ;$ Chi $^{2}=44.13, d f=15\left(P=0.0001 ; ; I^{2}=66 \%\right.$

Test for d 123

Navari et al, 2011

Shumwayet al, 2015

Navari et al, 2016 (sco

Navari et al, 2016 (NEIM)
Mukhopadhyay et al, 2017

Mukhopadhyay et al, 2017
Sapkota et al, 2017

Dulal et al, 2019

Saldanha et al, 2019

Hashimoto et, al, 2020

Ithimakin et al, 2020
Vimolchalao et al, 2020

Yeo et al, 2020
Subtotal
To5\% CI

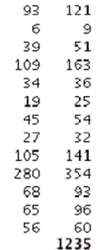

$\begin{array}{rrr}88 & 120 & 10.5 \% \\ 5 & 9 & 1.4 \% \\ 37 & 50 & 7.8 \% \\ 88 & 168 & 9.3 \% \\ 14 & 36 & 3.7 \% \\ 18 & 25 & 5.1 \% \\ 22 & 27 & 8.1 \% \\ 27 & 32 & 8.2 \% \\ 48 & 68 & 9.2 \% \\ 231 & 351 & 12.4 \% \\ 34 & 47 & 8.1 \% \\ 38 & 96 & 6.2 \% \\ 45 & 60 & 10.0 \% \\ & 1089 & 100.0 \%\end{array}$

$1.05[0.91,1.21]$
$1.20[0.57,2.53]$
$1.03[0.83,1.29]$
$1.28[1.07,1.5]$

$1.03[0.83,1.29]$

$2.43\{1.60,3.69\}$

$1.06[0.76,1.47]$
$1.02[0.82,1.27]$

$1.00[0.81,1.23]$

$1.2011 .10,1.321$

$1.01[0.81,1.25]$

$1.24[1.06,1.46]$
$1.17[1.06,1.28]$

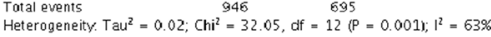

Test for overall effect: $Z=3.22$ (P $=0.00$,

1.2.3 MEC

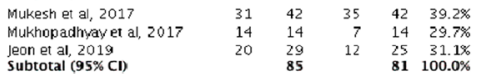

Tatal events
Heterogeneity Tau $^{2}=0.16 ; \mathrm{Chi}^{2}=10.00$, df $=2(\mathrm{P}=0.007) ;\left.\right|^{2}=80 \%$
Test for overall effect: $Z=0.99(\mathrm{P}=0.32)$

1.2.4 HEC/MEC

Mizukami et al, 2014

$\begin{array}{lllll}22 & 22 & 16 & 22 & 100.0 \% \\ 22 & & 22 & 100.0 \%\end{array}$

$1.36[1.05,1.77]$
$1.36[1.05,1.77]$

Total events
Heterogeneity Not applicable 22
Test for overall effect: $z=2.31$ ( $=0.021$

$\begin{array}{lllll}22 & 22 & & 22 & 100.0 \%\end{array}$

$0.89[0.71,1.11 \mid$

$1.44[0.89,2.31]$
$\mathbf{1 . 3 0}[0.78,2.17]$

1.2.5 Olanzapine $10 \mathrm{mg}$

Navari et al, 2011

Shumwayet al, 2015
Navari et al, 2016 (5CO)

Navari et al, 2016 (NEIM)

Mukesh et al, 2017

Mukhopadhyay et al, 2017

Sapkota et al, 2017

Celio e1 al, 2019

Dulal et at, 2019

Saldanha et al, 2019

Ithimakin et al, 2020
Vimolchalao et al, 2020

Vimolchalao et al, 2020
reo et al, 2020

rea et al, 2020
Subtotal $(95 \% \mathrm{CI}$

$\begin{array}{rr}93 & 121 \\ 8 & 9 \\ 39 & 51 \\ 109 & 163 \\ 31 & 42 \\ 48 & 50 \\ 19 & 25 \\ 45 & 54 \\ 27 & 32 \\ 20 & 29 \\ 105 & 141 \\ 33 & 46 \\ 65 & 96 \\ 56 & 60 \\ & 919\end{array}$

$\begin{array}{llll}88 & 120 & 9.6 \% & 1.05 \\ 5 & 0.91,1.211\end{array}$

$1.05[0.91,1.21]$
$1.20[0.57,2.53]$

$1.03[0.83,1.29 \mid$

$0.89\{0.71,1.11\}$

$2.29[1.64,3.18]$

$1.06[0.76,1.47]$

$1.02[0.82,1.27 \mid$

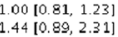

$1.05[0.88,1.26]$

$0.99[0.77,1.28]$
$1.71[1.29,2.27]$

$1.2411 .06,1.46$

Heterogeneity. Tau $^{2}=0.03 ; \mathrm{Chi}^{2}=42.36$, df $=13(\mathrm{P}<0.0001) ; \mathbf{l}^{2}=698$

Test for overall effect: $Z=2.57(P=0.01)$

1.2.6 Olanzapine $5 \mathrm{mg}$

$\begin{array}{lrrrrr}\text { Mizukami et al, } 2014 & 22 & 22 & 16 & 22 & \mathbf{1 4 . 7 \%} \\ \text { Hashimoto et al, 2020 } & 280 & 354 & 231 & 351 & 68.4 \% \\ \text { lthimakin et al 2020 } & 35 & 47 & 34 & 47 & 16.9 \% \\ \text { Subtotal (95\% CI) } & & 423 & & 420 & 100.9 \%\end{array}$

Heterogeneity, $\operatorname{Tau}^{2}=0.00 ; \mathrm{Chi}^{3}=2.43, \mathrm{df}=2(\beta=0.30) ; \mathrm{l}^{2}=18$

Test for overall effect: $z-3.23$ \& -0.001

1.2.7 Double-Blind Placebo-Controlled Design

Mizukami et al, 2014

Jeon et al, 2019

Hashimoto et al, 2020

thimakin et al, 2020

Yeo et al, 2020
Subtotal
To5\%

$\begin{array}{lrrrr}22 & 22 & 15 & 22 & 10.0 \% \\ 109 & 163 & 88 & 168 & 16.6 \%\end{array}$

$\begin{array}{rrrrr}22 & 22 & 16 & 22 & 10.0 \% \\ 20 & 163 & 88 & 168 & 16.6 \% \\ 280 & 354 & 12 & 25 & 3.7 \% \\ 68 & 354 & 351 & 28.7 \%\end{array}$

$\begin{array}{rrrrr}68 & 93 & 34 & 47 & 13.3 \% \\ 65 & 96 & 38 & 96 & 9.0 \% \\ 58 & 60 & 45 & 60 & 18.78 \% \\ & 817 & & 769 & 100.0 \%\end{array}$

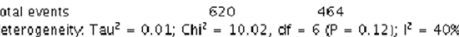

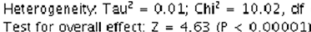

1.2.8 Open Controlled Design (Control Arm = All Antiemetics, Except Olanzapine)

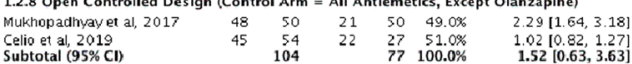
Subtotal (95\%

Tatal events
Heterogeneity. Tau $^{2}=0.38, \mathrm{Chi}^{2}=19.56, \mathrm{df}=1(\mathrm{P}<0.00001) ; \mathrm{I}^{2}=95 \%$

Heterogeneity Tau $^{2}=0.38$, Chi $^{2}=19.56$
Test for overall effect: $Z=0.93\langle P=0.35\}$

1.2.9 Open Controlled Design (Control Arm = Antiemetics Not In Olanzapine Arm)

$1.05[0.91,1.2$
$1.20[0.57,2.5$

Shumway et al, 2015

$60 \mathrm{sco}$

Mukesh et al, 2017

Dullal et al, 2019

Saldanha et al, $2019 \mathrm{~s}$
Subtotal $(95 \% \mathrm{Cl})$

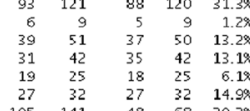

Total

$\begin{array}{rrrrr}27 & 32 & 27 & 32 & 14.9 \% \\ 105 & 141 & 48 & 68 & 20.2 \%\end{array}$

320

258

Heterogeneity $\mathrm{Tau}^{2}=0.00 ; \mathrm{Chi}^{2}=2.07$, df
Test for overall effect: $Z=0.47\langle\mathrm{P}=0.64\rangle$

$1.36[1.05,1.77]$ $1.20[1.10,1.32]$
$1.03[0.81,1.31]$
$1.19[1.07,1.33]$

$1.36[1.05,1.77]$ $1.28[1.07,1.53]$ $.44[0.89,2.31]$
$.20 \mid 1.10,1.32]$ $1.01[0.81,1.25]$ $1.24[1.06,1.46]$
$1.26[1.14,1.38]$

Fig. 1 (continued)

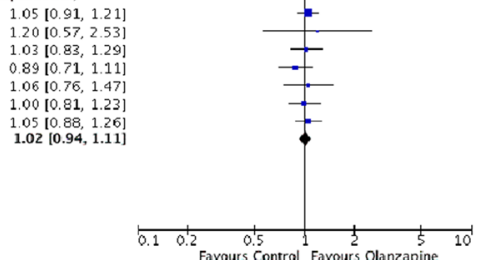


1.3

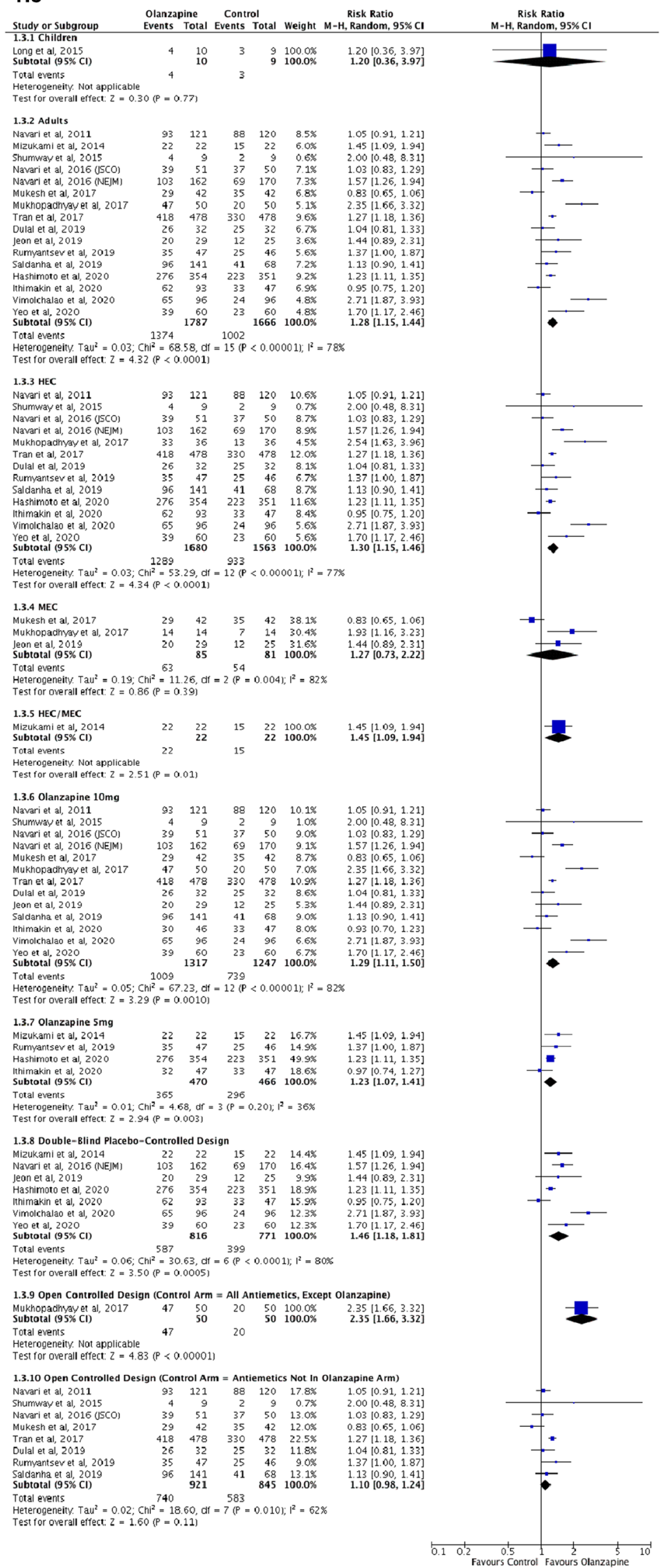

Fig. 1 (continued) 
Table 2 Absolute risk difference between olanzapine and other regimens for statistically significant differences

\begin{tabular}{|c|c|c|}
\hline Endpoint & $\begin{array}{l}\text { Risk difference }(95 \% \\
\text { confidence interval) }\end{array}$ & $\begin{array}{l}\text { Clinically } \\
\text { significant? }\end{array}$ \\
\hline Complete response, acute phase - adults & $0.10(0.05,0.15)$ & Yes \\
\hline Complete response, acute phase - HEC & $0.11(0.05,0.17)$ & Yes \\
\hline Complete response, acute phase - olanzapine $10 \mathrm{mg}$ & $0.10(0.04,0.17)$ & Yes \\
\hline $\begin{array}{l}\text { Complete response, acute phase - double-blind placebo-controlled } \\
\text { design }\end{array}$ & $0.17(0.07,0.27)$ & Yes \\
\hline $\begin{array}{l}\text { Complete response, acute phase - open controlled design (control } \\
\text { arm = antiemetics not in olanzapine arm) }\end{array}$ & $0.06(0.01,0.11)$ & No \\
\hline Complete response, delayed phase - adults & $0.12(0.05,0.20)$ & Yes \\
\hline Complete response, delayed phase - HEC & $0.12(0.05,0.20)$ & Yes \\
\hline Complete response, delayed phase - olanzapine $10 \mathrm{mg}$ & $0.11(0.02,0.20)$ & Yes \\
\hline Complete response, delayed phase - olanzapine $5 \mathrm{mg}$ & $0.14(0.03,0.24)$ & Yes \\
\hline $\begin{array}{l}\text { Complete response, delayed phase - double-blind } \\
\text { placebo-controlled design }\end{array}$ & $0.16(0.10,0.22)$ & Yes \\
\hline Complete response, overall phase - adults & $0.17(0.10,0.24)$ & Yes \\
\hline Complete response, overall phase - HEC & $0.18(0.10,0.25)$ & Yes \\
\hline Complete response, overall phase - olanzapine $10 \mathrm{mg}$ & $0.16(0.07,0.25)$ & Yes \\
\hline Complete response, overall phase - olanzapine $5 \mathrm{mg}$ & $0.15(0.04,0.26)$ & Yes \\
\hline $\begin{array}{l}\text { Complete response, overall phase - double-blind placebo-controlled } \\
\text { design }\end{array}$ & $0.22(0.12,0.33)$ & Yes \\
\hline No nausea, acute phase - adults & $0.13(0.07,0.19)$ & Yes \\
\hline No nausea, acute phase - HEC & $0.14(0.06,0.21)$ & Yes \\
\hline No nausea, acute phase - olanzapine $10 \mathrm{mg}$ & $0.14(0.07,0.20)$ & Yes \\
\hline No nausea, acute phase - double-blind placebo-controlled design & $0.26(0.19,0.33)$ & Yes \\
\hline No nausea, delayed phase - adults & $0.19(0.12,0.26)$ & Yes \\
\hline No nausea, delayed phase - HEC & $0.19(0.11,0.26)$ & Yes \\
\hline No nausea, delayed phase - olanzapine $10 \mathrm{mg}$ & $0.19(0.12,0.26)$ & Yes \\
\hline No nausea, delayed phase - double-blind placebo-controlled design & $0.19(0.11,0.27)$ & Yes \\
\hline $\begin{array}{l}\text { No nausea, delayed phase - open controlled design }(\text { control arm }= \\
\text { antiemetics not in olanzapine arm) }\end{array}$ & $0.16(0.03,0.28)$ & Yes \\
\hline No nausea, overall phase - adults & $0.20(0.13,0.26)$ & Yes \\
\hline No nausea, overall phase - HEC & $0.21(0.14,0.28)$ & Yes \\
\hline No nausea, overall phase - olanzapine $10 \mathrm{mg}$ & $0.20(0.13,0.27)$ & Yes \\
\hline No nausea, overall phase - double-blind placebo-controlled design & $0.20(0.11,0.29)$ & Yes \\
\hline $\begin{array}{l}\text { No nausea, overall phase - open controlled design }(\text { control arm }= \\
\text { antiemetics not in olanzapine arm) }\end{array}$ & $0.15(0.05,0.26)$ & Yes \\
\hline No emesis, delayed phase - adults & $0.20(0.13,0.26)$ & Yes \\
\hline No emesis, delayed phase - HEC & $0.17(0.09,0.25)$ & Yes \\
\hline No emesis, delayed phase - olanzapine $10 \mathrm{mg}$ & $0.20(0.13,0.26)$ & Yes \\
\hline No emesis, overall phase - adults & $0.19(0.11,0.28)$ & Yes \\
\hline No emesis, overall phase - HEC & $0.25(0.13,0.37)$ & Yes \\
\hline No emesis, overall phase - olanzapine $10 \mathrm{mg}$ & $0.19(0.11,0.28)$ & Yes \\
\hline
\end{tabular}

review summarizes the results across 25 studies, which reported on 4275 patients. One study reported the effect of olanzapine on children, and three studies reported on olanzapine for the rescue of breakthrough CINV; the remaining 23 studies reported on olanzapine for the prophylaxis of CINV in adults, across 4217 patients. Zhou et al. reported on acute and delayed emetic control with or without nausea control, Bahbah et al. meta-analyzed complete response and nausea control rates, and Sutherland et al. summarized instances where patients successfully experienced no nausea and no emesis; our review reports on complete response, nausea control, and emetic control.

Fig. 2 Efficacy of olanzapine regimens compared to others for the prophylaxis of chemotherapy-induced nausea and vomiting (CINV)no nausea. 2.1 Acute phase. 2.2 Delayed phase. 2.3 Overall phase 
2.1

\begin{tabular}{|c|c|c|c|c|c|c|}
\hline \multirow{2}{*}{ Subgroup } & \multicolumn{2}{|c|}{ Olanzapine } & \multicolumn{2}{|c|}{ Control } & \multirow[b]{2}{*}{ Weight } & \multirow{2}{*}{$\begin{array}{c}\text { Risk Ratio } \\
\text { M-H, Random, } 95 \% \mathrm{Cl}\end{array}$} \\
\hline & Events & Total & Events & Total & & \\
\hline 2.1.1 & & & & & & \\
\hline Tan et al, 2009 & 117 & 121 & 98 & 108 & $10.4 \%$ & $1.07[0.99,1.1$ \\
\hline Navari et al, 2011 & 105 & 121 & 104 & 120 & $10.0 \%$ & $1.00[0.91,1.11$ \\
\hline Shumway et al, 2015 & 3 & 9 & 5 & 10 & $1.0 \%$ & $0.67[0.22,2.0$ \\
\hline Whang et al, 2015 & 28 & 42 & 19 & 42 & $4.8 \%$ & $1.47[0.99,2.1$ \\
\hline Navari et al, 2016 (JSCO) & 44 & 51 & 39 & 50 & $8.5 \%$ & $1.11[0.92,1]$ \\
\hline Navari et al, 2016 (NEJM) & 135 & 183 & 82 & 181 & $8.5 \%$ & $1.63[1.36$, \\
\hline Mukesh et al, 2017 & 35 & 42 & 29 & 42 & $7.3 \%$ & 1.21 \\
\hline Mukhopadhyayet a & 48 & 50 & 43 & 50 & $9.6 \%$ & 1.12 \\
\hline Celio et al, 2019 & 46 & 54 & 21 & 27 & $7.5 \%$ & $1.10[0.87$, \\
\hline Dulal et al, 2019 & 27 & 32 & 26 & 32 & $7.7 \%$ & $1.04[0.83$, \\
\hline Saldanha et al, 2018 & 98 & 141 & 43 & 68 & 7.986 & $1.10[0.89$ \\
\hline Tienchaiananda et al, 2019 & 10 & 20 & 2 & 19 & $0.7 \%$ & $4.75[1.19,18$ \\
\hline thimakin et al, 2020 & 52 & 93 & 21 & 47 & $5.2 \%$ & $1.25[0.87]$, \\
\hline Vimalchalao et al, 2020 & 48 & 96 & 21 & 96 & $4.3 \%$ & $2.29[1.49,3$ \\
\hline rea et al, 2020 & 46 & 60 & 32 & 60 & $6.7 \%$ & $1.44[1.09$ \\
\hline $\begin{array}{l}\text { Subtotal }(95 \\
\text { Total events }\end{array}$ & & & & & & \\
\hline
\end{tabular}

Total events
Heterogeneity. Tau $^{2}=0.03 ; \mathrm{Chi}^{2}=672 \quad 58.46, \mathrm{df}=14(\mathrm{P}<0.00001) ; \mathrm{l}^{2}=79 \%$

Heterogeneity. $\operatorname{Ta}^{2}=0.03 ; \mathrm{Chi}^{2}=67.46,0$
Test for overall effect: $Z=3.31(P=0.0009)$

2.1.2 HEC

Tan et al, 2009

Navari et al, 2011
Shumwayet al, 2015

Shumway et al, 2015
Wiang et al, 2015

Navari et al, 2016 (JSC)

Navari et al, 2016 (NEJM)

Celio et al, 2019

Dulal et al, 2019
Saldanha et al, 2019

Saldanha et al, 20199
Tienchaiananda et al, 2019

trhimakin et al, 2020

Vimalchalao et al, 2020
Yeo et al, 2020
Subtotal $(95 \% \mathrm{Cl})$

Total events
Heterogeneity. Tau $^{2}=0.04 ; \mathrm{Chi}^{2}=57.27$, df $=12(\mathrm{P}<0.00001) ; \mathrm{l}^{2}=79 \%$

$\begin{array}{rrrrrr}53 & 56 & 40 & \mathbf{4 6} & 11.0 \% & 1.09[0.96,1.24] \\ 105 & 121 & 104 & \mathbf{1 2 0} & 11.4 \% & 1.00[0.91,1.11] \\ 3 & 9 & 5 & 10 & 1.5 \% & 0.67[0.22,2.03] \\ 28 & 42 & 19 & \mathbf{4 2} & 6.3 \% & 1.47[0.99,2.19] \\ 44 & 51 & 39 & 50 & 10.0 \% & 1.11[0.92,1.33] \\ 135 & 183 & 82 & \mathbf{1 8 1} & 10.1 \% & 1.63[1.36,1.93] \\ 46 & 54 & 21 & 27 & 9.2 \% & 1.10[0.87,1.38] \\ 27 & 32 & 26 & 32 & 9.3 \% & 1.04[0.83,1.30] \\ 98 & 141 & 43 & 68 & 9.5 \% & 1.10[0.89,1.36] \\ 10 & 20 & 2 & 19 & 1.0 \% & 4.75[1.19,18.92] \\ 52 & 93 & 21 & \mathbf{4 7} & 6.7 \% & 1.25[0.87,1.80] \\ 48 & 96 & 21 & 96 & 5.8 \% & 2.29[1.49,3.51] \\ 46 & 60 & 32 & 66 & 8.3 \% & 1.44[1.09,1.89] \\ & 958 & & 798 & \mathbf{1 0 0 . 0 \%} & 1.24[1.08,1.43]\end{array}$

Heterogeneity. $\mathrm{Tau}^{2}=0.04 ; \mathrm{Chi}^{2}=57.27$,
Test for overall effect: $Z=2.96(P=0.003)$

2.1.3 MEC

Tan et al, 2009

$\begin{array}{llrrrr}\text { Mukesh et al, } 2017 & 35 & 42 & 29 & 42 & 30.6 \% \\ \text { Subtotal }(95 \% \mathrm{Cl}) & & 107 & & 104 & 100.0 \%\end{array}$

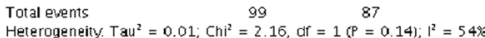

Heterogeneity. $\operatorname{Tau}^{2}=0.01 ; \mathrm{Chi}^{2}=2.16$, df
Test for overall effect: $Z=1.06(P=0.29)$

2.1.4 HEC/MEC

\begin{tabular}{|c|c|c|c|c|c|c|}
\hline $\begin{array}{l}\text { Muknopadhyayet al, } 2017 \\
\text { Subtotal }(95 \% \mathrm{Cl})\end{array}$ & 48 & $\begin{array}{l}50 \\
50\end{array}$ & 43 & $\begin{array}{l}50 \\
50\end{array}$ & $\begin{array}{l}100.0 \% \\
100.0 \%\end{array}$ & $\begin{array}{l}1.12[0.98,1.27] \\
1.12[0.98,1.27]\end{array}$ \\
\hline \multicolumn{7}{|c|}{$\begin{array}{l}\text { Heterogeneity. Not applicable } \\
\text { Test for overal effect: } Z=1.72(P=0.09)\end{array}$} \\
\hline \multicolumn{7}{|l|}{2.1 .5 Olanzapine $10 \mathrm{mg}$} \\
\hline Tan et al, 2009 & 117 & 121 & 98 & 108 & $10.3 \%$ & $1.07[0.99,1.14]$ \\
\hline Navari et al, 2011 & 105 & 121 & 104 & 120 & $9.9 \%$ & $1.00[0.91,1.11]$ \\
\hline Shumwayet al, 2015 & 3 & 9 & 5 & 10 & $1.0 \%$ & $0.67[0.22,2.03]$ \\
\hline Wwang et al, 2015 & 28 & 42 & 19 & 42 & $4.8 \%$ & $1.47[0.99,2.19]$ \\
\hline Navari et al, $2016(\mid s \mathrm{CO})$ & 44 & 51 & 39 & 50 & $8.5 \%$ & $1.11[0.92,1.33]$ \\
\hline Navari et al, 2016 (NEJM) & 135 & 183 & 82 & 181 & $8.5 \%$ & $1.63[1.36,1.95]$ \\
\hline Mukesh et al 2017 & 35 & 42 & 29 & 42 & $7.3 \%$ & $121[095,154]$ \\
\hline Mukhopadhyavet al, 2017 & 48 & 50 & 43 & 50 & $9.5 \%$ & $1.12[0.98,1.27]$ \\
\hline Celio et al, 2019 & 46 & 54 & 21 & 27 & $7.6 \%$ & $1.10[0.87,1.38]$ \\
\hline Dulal et al, 2019 & 27 & 32 & 26 & 32 & $7.7 \%$ & $1.04[0.83,1.30]$ \\
\hline Saldanha et al, 2019 & 98 & 141 & 43 & 68 & $7.9 \%$ & $1.10[0.89,1.36]$ \\
\hline Tienchaiananda et al, 2019 & 10 & 20 & 2 & 19 & $0.7 \%$ & $4.75[1.19,18.92]$ \\
\hline thimakin et al, 2020 & 30 & 46 & 21 & 47 & $5.0 \%$ & $1.46[1.00,2.14]$ \\
\hline Vimolchalan et al, 2020 & 48 & 86 & 21 & $\Leftrightarrow 6$ & $4.4 \%$ & $2.29[1.48,3.51]$ \\
\hline Yeo et al, 2020 & 46 & 50 & 32 & 60 & $6.7 \%$ & $1.44[1.09,1.89]$ \\
\hline Subtotal $(95 \% \mathrm{Cl})$ & & 1068 & & 952 & $100.0 \%$ & $1.23[1.09,1.38]$ \\
\hline
\end{tabular}

Subtotal $(95 \% \mathrm{Cl})$

820

$1.05[0.98,1.13]$

$1.21[0.95,1.54]$
$1.10[0.92,1.30]$

Heterogeneity Tau $^{2}=0.03 ; \mathrm{Ch}^{2}=70.61$, of $=14(\mathrm{P}<0.00001) ; \mathrm{l}^{2}=808$

2.1.6 Olanzapine $5 \mathrm{mg}$

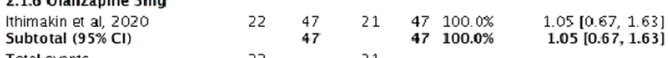

Total events

Heterogeneity Not applicable

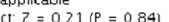

2.1.7 Double-Blind Placebo-Controlled Design $\begin{array}{lrrrrr}\text { Navari et al, } 2016 \text { (NEJM) } & 135 & 183 & 82 & 181 & 34.9 \% \\ \text { Tienchaiananda et al, 2019 } & 10 & 20 & 2 & 19 & 2.3 \%\end{array}$

thimakin et al, 2020

Vimalchalao et al, 2020

Yea et al, 2020

$\begin{array}{rrrrr}135 & 183 & 82 & 181 & 34.9 \% \\ 10 & 20 & 2 & 19 & 2.3 \% \\ 52 & 93 & 21 & 47 & 19.9 \% \\ 48 & 96 & 21 & 96 & 16.5 \% \\ 46 & 50 & 32 & 60 & 26.5 \% \\ & 452 & & 403 & \mathbf{1 0 0 . 0 \%}\end{array}$

Total events

$291 \quad 158 \quad 403100.0 \%$

Heterogeneity. Tau $^{2}=0.03 ; \mathrm{Chi}^{2}=7.66$, df
Test for overall effect: $Z=4.38(\mathrm{P}<0.0001)$

2.1.8 Open Controlled Design (Control Arm = All Antiemetics, Except Olanzapine)

Tan et al, 20099

Wukhopadhyay et al, 2017

$\begin{array}{rrrrr}117 & 121 & 98 & 108 & 55.8 \% \\ 28 & 42 & 19 & 42 & 4.1 \%\end{array}$

Cellio et al, 2019
Subtotal (95\% CI)

Total event

$\begin{array}{rrrrrr}117 & 121 & 98 & 108 & 55.8 \% & 1.07[0.99,1.14] \\ 28 & 42 & 19 & 42 & 4.1 \% & 1.47[0.99,2.19] \\ 48 & 50 & 43 & 50 & 29.1 \% & 1.12[0.98,1.27]\end{array}$

$1.63[1.36,1.95]$

$4.75[1.19,18.92]$
$1.25[0.87,1.80]$

$2.29[1.49,3.51]$

$1.44[1.09,1.89]$
$1.62[1.31,2.01]$

239

\begin{tabular}{rr}
227 & 11.18 \\
\hline $200.0 \%$
\end{tabular}

$1.10[0.87,1.38]$

2.1.9 Open Controlled Design (Control Arm = Antiemetics Not In Olanzapine Arm)

Navari et al, 2011

Navari et al, 2016 USCO

Mukesh et al, 2017

Dulal et al, 2019

Subtotal $(95 \% \mathrm{CI})$

Total events

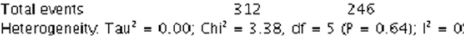

Test

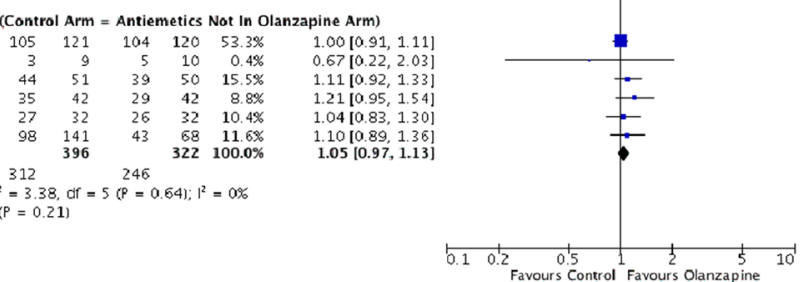


2.2

\begin{tabular}{|c|c|c|c|c|c|c|}
\hline \multirow{3}{*}{$\frac{\text { Study or Subgroup }}{2.2 .1 \text { Adults }}$} & \multicolumn{2}{|c|}{ Olanzapine } & \multicolumn{2}{|c|}{ Contral } & \multirow[b]{2}{*}{ Weight } & \multirow{2}{*}{$\begin{array}{c}\text { Risk Ratio } \\
\text { M-H, Rando m, } 95 \% \mathrm{CI}\end{array}$} \\
\hline & & & & & & \\
\hline & & & & & & \\
\hline Tan et al, 2009 & 93 & 121 & 50 & 108 & $9 \%$ & $1.66[1]$ \\
\hline & 83 & 121 & 45 & 120 & & \\
\hline 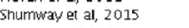 & 4 & 9 & 2 & 9 & & 2.000 \\
\hline w & 35 & 42 & 22 & 42 & & \\
\hline Navari et al, 2 & 36 & 51 & 20 & 50 & & \\
\hline Navari & 75 & 177 & 45 & 177 & $9 \%$ & 37 \\
\hline Mukes & 24 & 42 & 23 & 42 & $\%$ & 71,1 \\
\hline Mukno & 38 & 50 & 19 & 50 & & \\
\hline Celio & 32 & 54 & 20 & 27 & & $88,1$. \\
\hline Dulal & 22 & 32 & 24 & 32 & $7.9 \%$ & $.92[0.67,1.25$ \\
\hline Saldann & 98 & 141 & 40 & 88 & $8.9 \%$ & $1.18[0.94,1.48$ \\
\hline ida et al, 201 & 6 & 20 & 0 & 19 & $0.4 \%$ & $38[0.75,205.75]$ \\
\hline thima & 37 & 93 & 16 & 47 & $5.9 \%$ & $1.17[0.73,1.87]$ \\
\hline & 40 & 96 & 13 & 96 & $5.0 \%$ & $3.08[1.76$, \\
\hline & $4 \overline{6}$ & & 38 & & & \\
\hline Subtotal $(95 \% \mathrm{Cl})$ & & 1109 & & 947 & $100.0 \%$ & $1.42[1.20$ \\
\hline
\end{tabular}

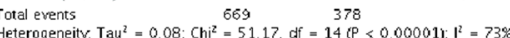

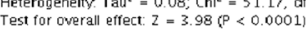

2.2.2 HEC

Tan et al, 2009

Navari et al, 2011

Shumway et al, 2015
Wang et al, 2015

Navari et al, 2015 (ISCO)

Navari et al, 2016 (NE)M)

Celio et al, 2019

Dulal et al, 2019

Tienchaiananda et al, 2019

Tienchaiananda et al, 2019
thimakin et al, 2020
Vimolchalao et al, 2020

Yeo et al, 2020
Subtotal $(95 \% \mathrm{Cl})$

$$
\begin{array}{rr}
39 & 56 \\
83 & 121 \\
4 & 9 \\
35 & 42 \\
36 & 51 \\
75 & 177 \\
32 & 54 \\
22 & 32 \\
98 & 141 \\
6 & 20 \\
37 & 93 \\
40 & 96 \\
46 & 60 \\
& 952
\end{array}
$$

$\begin{array}{rrrr}14 & 46 & 7.4 \% & 2.29[1.43,3.66] \\ 46 & 120 & 10.0 \% & 1.79[1.38,2.31]\end{array}$

$2.29[1.43,3.66]$
$1.79[1.38,2.31]$
$2.00[0.48,8.3]$

$2.00[0.48,8.31]$

$\begin{array}{ll}42 & 9.3 \% \\ 50 & 8.4 \%\end{array}$

$1.76[1.20,2.59]$

$1.67[1.23,2.26]$

$0.80[0.58,1.10]$

$0.92[0.67,1.25]$

$\begin{array}{rrrrrr}46 & 60 & 38 & 60 & 10.3 \% & 1.21[0.95,1.54]\end{array}$

Heterngeneity Tau $^{2}=0.09 ; \mathrm{Chi}^{2}=48.48$, df $=12\left(\mathrm{P}<0.000011 ; \mathrm{l}^{2}=75 \%\right.$

Test for overall effect $z=3.55 \%=0.0004$

2.2.3 MEC

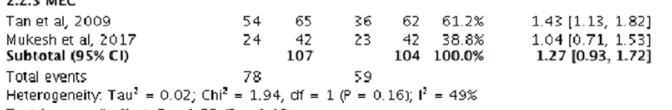

Test for overall effect $z=1.52(P=0.13)$

2.2.4 HEC/MEC

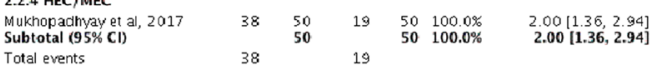

Heterogeneity: Not applicable
Test for overall effect $z=3.51(P=0.0004)$

Test for overall effect 2

Tan et al, 2009

Nevari et al, 2011
Shumway et al, 2015

Whang et al, 2015

Navari et al, 2016 JSCO)

Navari et al, 2018 (NEIM)

ukesh et al, 2017 , 2017

Celio et al, 2019

Dulal et al, 2019

Saldanha et al, 2019
Tienchaiananda et al, 2019

Tienchaiananda et al, 2019
thimakin et al, 2020

Vimakin et al, 2020

reo et al, 2020

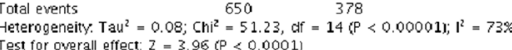

2.2.6 Olanzapine $5 \mathrm{mg}$

$\begin{array}{lllllll}\text { Thimakin et al, } 2020 & 19 & 47 & 15 & 47 & 100.0 \% & 1.19[0.70,2.01] \\ \text { Subtotal (95\% C1) } & & 47 & & 47 & 100.0 \% & \mathbf{1 . 1 9}[0.70,2.01]\end{array}$

Total events

19

$\begin{array}{llllll}93 & 121 & 50 & 108 & 9.0 \% & 1.66[1.33,2.08] \\ 83 & 121 & 46 & 120 & 8.6 \% & 1.79[1.38,2.31]\end{array}$

$\begin{array}{rrrrrrr}4 & 3 & 2 & 9 & 1.3 \% & 2.00[0.48,8.31] \\ 35 & 42 & 22 & 42 & 7.8 \% & 1.59[1.15,2.19]\end{array}$

$\begin{array}{lllll}51 & 22 & 42 & 7.8 \% & 1.59[1.15,2.199\end{array}$

$\begin{array}{lllllll}75 & 177 & 45 & 177 & 8.0 \% & 1.67[1.23,2.26]\end{array}$

$\begin{array}{llllll}24 & 42 & 23 & 42 & 7.0 \% & 1.04[0.71,1.53] \\ 38 & 50 & 19 & 50 & 7.0 \% & 2.00[1.36,2.94]\end{array}$

$\begin{array}{llllll}32 & 54 & 20 & 27 & 7.9 \% & 0.80[0.58,1.10] \\ 22 & 32 & 24 & 32 & 7.9 \% & 0.92[0.67,1.25]\end{array}$

$1.18[0.94,1.48$

$3810.75,205.75]$
$1.15[0.67,1.97]$

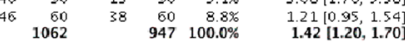

Test for overall effect: $z=0.64(P=0.52)$

2.2.7 Double- Blind Placebo-Controlled Design

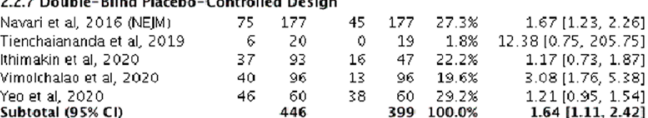

Yeo et al, 2020
Subtotal $(95 \% \mathrm{Cl})$

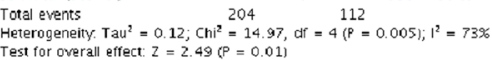

2.2.8 Open Controlled Design (Control Arm = All Antiemetics, Except Olanzapine)

$\begin{array}{lllllll}\text { tan et al, } 2009 & 93 & 121 & 50 & 108 & 27.2 \% & 1.66[1.33,2.08]\end{array}$

Mukhopadhyay et al, 2017

Celio et al, 2019
Subtotal $95 \% \mathrm{Cl}$

$\begin{array}{lllll}35 & 42 & 22 & 42 & 24.98 \\ 38 & 50 & 19 & 50 & 22.9 \%\end{array}$

$\begin{array}{lrrrr}32 & 54 & 20 & 27 & 25.0 \% \\ 267 & 227 & 100.0 \%\end{array}$

$1.5911 .16,2.19$

$0.80[0.58,1.10]$

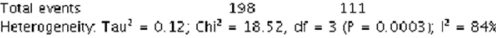

Test for overal effer $z=185$ o $P=0.06$

2.2.9 Open Controlled Design (Control Arm = Antiemetics Not In Olanzapine Arm)

Navari et al $2011 \quad 83 \quad 121 \quad 46 \quad 120 \quad 213 \%$

Navari et al, 2011

Navari et al, 2015 (15C

Mukesh et al, 2017

Dulal et al, 2019

Saldanha et al, 201

$\begin{array}{rrrrr}83 & 121 & 46 & 120 & 21.3 \\ 4 & 9 & 2 & 9 & 29 \pi\end{array}$

$1.79[1.38,2.31]$

$.00[0.48,8.31]$
$1.76[1.20,2.59]$

$\begin{array}{llllll}38 & 51 & 20 & 50 & 16.9 \% & 1.76[1.20,2.59 \\ 24 & 42 & 23 & 42 & 17.0 \% & 1.04[0.71,1.53] \\ 22 & 32 & 24 & 32 & 19.5 \% & 0.92[0.67,1.25]\end{array}$

$1.18[0.94,1.48]$
$\mathbf{1 . 3 1}[\mathbf{1 . 0 1}, \mathbf{1 . 6 9}]$

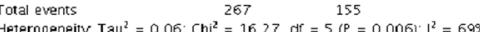

Test for overall effect $z=2.03 \mathrm{ip}=0.04$

Risk Ratio

M-H, Random, $95 \% \mathrm{CI}$

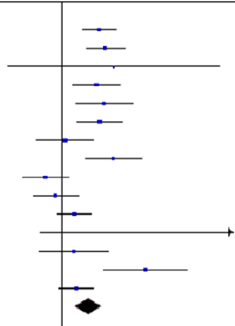

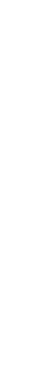

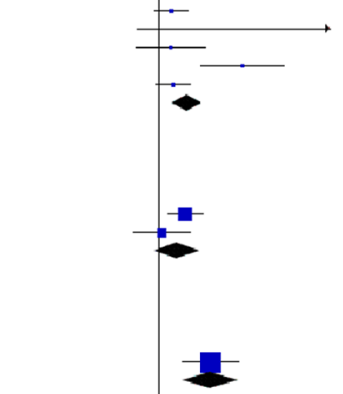

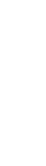

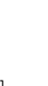

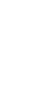

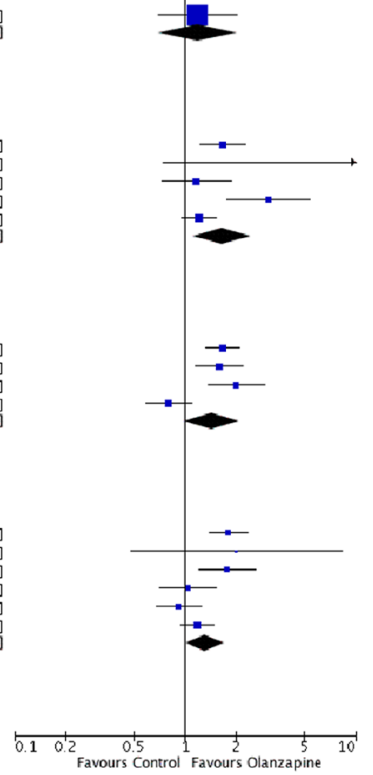

Fig. 2 (continued) 
2.3

\begin{tabular}{|c|c|c|c|c|c|c|}
\hline \multirow[b]{2}{*}{ Study or Subgroup } & \multicolumn{2}{|c|}{ Olanzapine } & \multicolumn{2}{|c|}{ Contro } & \multirow[b]{2}{*}{ Weight } & \multirow{2}{*}{$\begin{array}{c}\text { Risk Ratio } \\
\text { M-H, Random, } 95 \% \mathrm{Cl}\end{array}$} \\
\hline & & Total & & Total & & \\
\hline 2.3.1 Adults & & & & & & \\
\hline Tan et al, 2009 & 93 & 121 & 48 & 108 & $11.9 \%$ & $1.73[1.37,2.18]$ \\
\hline Navari et al, 2011 & 83 & 121 & 48 & 120 & $11.4 \%$ & $1.79[1.38,2.31]$ \\
\hline Shumway et al, 2015 & 2 & 9 & 3 & 10 & $1.3 \%$ & $0.74[0.18,3.48]$ \\
\hline Navari et al, 2015 gJScol & 36 & 51 & 21 & 50 & $9.2 \%$ & $1.68[1.15,2.44]$ \\
\hline Navari et al, 2016 (NEJM) & 66 & 177 & 39 & 178 & $9.8 \%$ & $1.70[1.22,2.38]$ \\
\hline Mukes & 23 & 42 & 23 & 42 & $8.8 \%$ & $1.00[0.68,1.48]$ \\
\hline Dulal et al, 2019 & 22 & 32 & 23 & 32 & $10.2 \%$ & $0.96[0.70,1.32]$ \\
\hline Rumyantsevet al, 2019 & 21 & 47 & 11 & $4 \overline{6}$ & $5.6 \%$ & $1.87[1.02,3.42]$ \\
\hline Saldanha et al, 2019 & 89 & 141 & 33 & 68 & $11.1 \%$ & $1.30[0.99,1.71]$ \\
\hline Tienchaiananda et al, 2019 & 6 & 20 & 0 & 19 & $0.4 \%$ & $12.38[0.75,205.75]$ \\
\hline Ithimakin et al, 2020 & 30 & 93 & 13 & 47 & $6.3 \%$ & $1.17[0.67,2.02]$ \\
\hline Yimolchalao et al, 2020 & 39 & 96 & 11 & 96 & $5.6 \%$ & $3.55[1.93,6.50]$ \\
\hline Yeo et & 35 & & 20 & 60 & $8.3 \%$ & $1.75[1.15,2.66]$ \\
\hline Subtotal $(95 \% \mathrm{Cl})$ & & 1010 & & 876 & $100.0 \%$ & $1.53[1.28,1.84]$ \\
\hline
\end{tabular}

Yeo et al, 2020
Subtotal
$(95 \% \mathrm{Cl})$

Tatal events 545 $545 \quad 291$ $876 \quad 100.0 \%$

$1.53[1.28,1.84]$

Heterogeneity Tau $^{2}=0.06 ; \mathrm{Chi}^{2}=30.63, \mathrm{df}=12(\mathrm{P}=0.002) ; \mathrm{I}^{2}=61 \%$

Test for overall effect: $z=4.57$ (P<0.00001)

2.3.2 HEC

Tan et al, 2009

Navari et al, 2011

Shumwayet al, 2015

Navari at al, 2016 d JSCOI

Navari et al, 2016 (NEJM)

Dulal et al, 2019

Rumyant sev et al, 2019

Saldanha et al, 2019

Tienchaiananda et al, 2019

Ithimakin et al, 2020

Vimolchalao et al, 2020

Yeo et al, 2020
Subtotal $(95 \% \mathrm{CI})$

Tatal events
Heterogeneity. $\mathrm{Tau}^{2}=0.07 ; \mathrm{Chi}^{2}=28.74, \mathrm{di}=11(\mathrm{P}=0.002) ; \mathrm{I}^{2}=62 \%$

$\begin{array}{rrrrr}39 & 56 & 13 & 46 & 8.4 \% \\ 83 & 121 & 46 & 120 & 12.8 \% \\ 2 & 9 & 3 & 10 & 1.6 \% \\ 36 & 51 & 21 & 50 & 10.6 \%\end{array}$

$\begin{array}{rrrrr}66 & 177 & 39 & 178 & 11.2 \% \\ 22 & 32 & 23 & 32 & 116 \%\end{array}$

$\begin{array}{rrrrr}21 & 47 & 11 & 46 & 5.8 \% \\ 89 & 141 & 33 & 68 & 12.4 \%\end{array}$

$\begin{array}{rr}89 & 141 \\ 6 & 20\end{array}$

$368 \quad 12.4 \%$

$1.87[1.02,3.42]$

$1.30[0.99,1.71]$

$2.46[1.51,403]$ $79[1.38,2.31]$

(6., 3.48]

$1.68[1.16,2.44]$

$1.70[1.22,2.38]$

$\begin{array}{llllrr}30 & 93 & 13 & 47 & 7.5 \% & 12.38[0.75,205.75] \\ 39 & 96 & 11 & 96 & 6.8 \% & 3.17[0.67,2.02] \\ 35 & 6 & 20 & 69 & 9.9 \% & 1.55]\end{array}$

$55[1.93,6.50]$

Test for overall effect: $z=4.64(\mathrm{P}<0.00001)$

\subsubsection{MEC}

Tan

Mukesh et al, 2017

Subtotal $(95 \% \mathrm{C})$

$\begin{array}{rrrrr}54 & 65 & 35 & 62 & 57.8 \% \\ 23 & 42 & 23 & 42 & 42.2 \%\end{array}$

$1.64[1.33,2.02]$

atal events

$\begin{array}{lll}404 & 42.2 \%\end{array}$

$1.47[1.15,1.88]$ $1.00[0.68,1.48]$
$1.25[0.86,1.82]$

Heterogeneity Tau $^{2}=0.05: \mathrm{Chi}^{2}=2.77, \mathrm{~d}=1(\mathrm{P}=0.10) ; \mathrm{l}^{2}=648$

Test for overall effect: $z=1.16(\mathrm{P}=0.25)$

\subsubsection{Olanzapine $10 \mathrm{mg}$}

Tan et al, 2009

Navari et al, 2011

Narwari et al, 2016 clscol

Navari et al, 2016 ? JSCO

Navari te al, 2016 (NE)

Dulal et al, 2019

Saldanna et al, 201

Tiencha ananda et al, 2019

thimakin et al, 2020

Vimolchalao et al, 2020

Yeo et al, 2020

Subtotal $(95 \%$

$\begin{array}{rr}93 & 121 \\ 83 & 121 \\ 2 & 9 \\ 36 & 51 \\ 66 & 177 \\ 23 & 42 \\ 22 & 32 \\ 89 & 141 \\ 6 & 20 \\ 16 & 46 \\ 39 & 96 \\ 35 & 60 \\ & 916\end{array}$

$\begin{array}{rr}121 & 48 \\ 121 & 46 \\ 9 & 3 \\ 51 & 21 \\ 177 & 39 \\ 42 & 23 \\ 32 & 23 \\ 141 & 33 \\ 20 & 0 \\ 46 & 13 \\ 96 & 11 \\ 60 & 20 \\ 916 & \end{array}$

$108 \quad 12.6 \%$

$\begin{array}{rr}120 & 12.1 \% \\ 10 & 1.4 \%\end{array}$

$\begin{array}{rr}50 & 9.8 \% \\ 178 & 10.5 \%\end{array}$

$\begin{array}{rr}178 & 10.5 \% \\ 42 & 9.5 \%\end{array}$

$32 \quad 10.9 \%$

$19 \quad 0.4 \% \quad 12.38[0.75,205.75]$

$1.73[1.37,2.18]$

$79[1.38,2.31]$

.

$1.70[1,22,238$

$1.0010 .08,1.48$

$30[0.99,1.71$

$.38[0.75,205.75]$

$1.26[0.68,2.31]$

Heterogeneity. $\mathrm{Tau}^{2}=0.06: \mathrm{Chi}^{2}=29.72, \mathrm{df}=11(\mathrm{P}=0.0022) \mathrm{L}^{2}=63$

Test for overall effect: $z=4.31$ P $<0.00011$

2.3.5 Olanzapine $5 \mathrm{mg}$

$\begin{array}{lllllll}\text { Rumyantsevet al, } 2019 & 21 & 47 & 11 & 46 & 51.7 \% & 1.87 \\ {[1.02,3.42]}\end{array}$ $\begin{array}{lllllll}\text { Ithimakin et al, 2020 } & 14 & \mathbf{4 7} & 13 & \mathbf{4 7} & \mathbf{4 8 . 3 \%} & 1.08[0.57,2.04] \\ \text { Subtotal }(95 \% \mathbf{C l}) & & \mathbf{9 4} & & \mathbf{9 3} & \mathbf{1 0 0 . 0 \%} & \mathbf{1 . 4 3}[0.83,2.46]\end{array}$

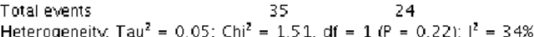

Heterogeneity $\mathrm{Tau}^{2}=0.05 ; \mathrm{Chi}^{2}=1.51, \mathrm{df}=1(\mathrm{P}=0.22) ; \mathrm{I}^{2}=34 \%$

Test for owerall effect: $Z=1.30(\mathrm{P}=0.19)$

2.3.6 Double-Blind Placebo-Controlled Design

Navari et al, 2016 (NE) M

Tienchaiananda et al, 2019

Ithimakin et al, 2020

Vimolchalao et al, 2020

Yeo et al, 2020

Subtotal $(95 \%$

Tatal events $176 \quad 83 \quad 05 \mathrm{~L}^{2}=57 \%$

$\begin{array}{ccccc}66 & 177 & 39 & 178 & 30.1 \%\end{array}$

\begin{tabular}{rrrr}
178 & $30.1 \%$ & $1.70[1.22,2.38]$ \\
\hline & 19 & $1.8 \%$ & $12.38[0.75,205.75]$
\end{tabular}

$2.38[0.75,205.75]$

$1.17[0.67,2.02]$

$3.55[1.93,6.50]$

$1.89[1.29,2.77]$

Teerogenert. $\mathrm{Tau}^{2}=0.10, \mathrm{Ch}^{2}=9.38$,

2.3.7 Open Controlled Design (Control Arm = All Antiemetics, Except Olanzapine)

$\begin{array}{lllllll}\text { Tan et al, } 2009 & 93 & 121 & 48 & 108 & 100.0 \% & 1.73[1.37,2.18] \\ \text { Subtotal }(95 \% \mathrm{Cl}) & & \mathbf{1 2 1} & & \mathbf{1 0 8} & \mathbf{1 0 0 . 0 \%} & \mathbf{1 . 7 3}[\mathbf{1 . 3 7}, \mathbf{2 . 1 8}] \\ \text { Total events } & 93 & & 48 & & & \end{array}$

Total events

Test for owerall effect: $z=4.62$ (P) $<0.00001)$

2.3.8 Open Controlled Design (Control Arm $=$ Antiemetics Not in Olanzapine Arm)

Navari et al, 2011

Shumway at al, 2015

Navari et al, 2016 J JSCO!

Mukesh et al, 2017

Rulal et al, 2019

Tumansevet al, 2019

Saldanha et al, 2019
Subtotal $(95 \% \mathrm{Cl})$

Total events

$\begin{array}{rrrrr}83 & 121 & 46 & 120 & 20.2 \% \\ 2 & 9 & 3 & 10 & 2.1 \% \\ 36 & 51 & 21 & 50 & 15.9 \% \\ 23 & 42 & 23 & 42 & 15.3 \% \\ 22 & 32 & 23 & 32 & 17.8 \% \\ 21 & 47 & 11 & 46 & 9.4 \% \\ 89 & 141 & 33 & 68 & 19.4 \% \\ & 443 & & 368 & 100.0 \%\end{array}$

276

160

160

Heterogeneity. $\mathrm{Tau}^{2}=0.05 ; \mathrm{Chi}^{2}=14.80$,
Test for owerall effect: $\mathrm{Z}=2.51(\mathbf{P}=0.01)$

$1.79[1.38,2.31]$

$0.74[0.16,3.48]$

$1.00[0.68,1.48]$

$0.96[070,132]$

$1.96[0.70,1.32]$

$1.30[0.99,1.71]$

Random, 95\% C
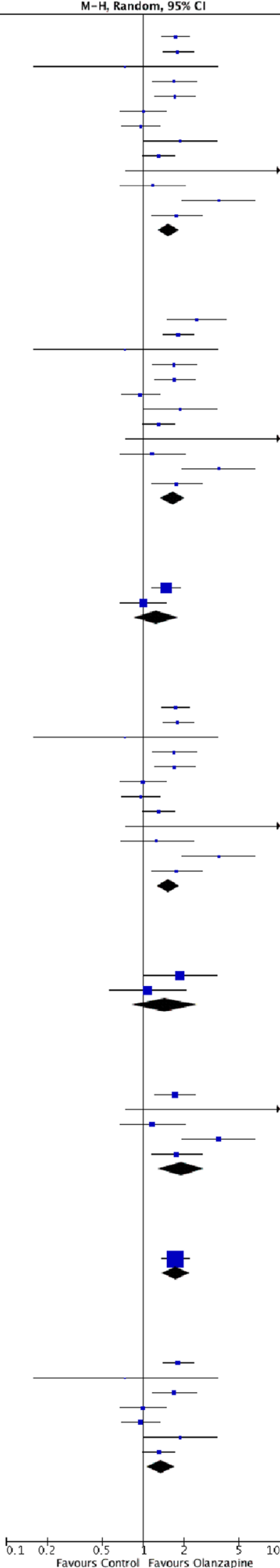

Fig. 2 (continued) 
3.1

Olanzapine Control Risk Ratio

Events Total Events Total Weight M-H, Random, $95 \% \mathrm{CI}$

Study or Subgroup

3.1.1 Adults

Tan et al, 2009

Shumway et al, 2015

wang et al, 2015

Yeo et al, 2020

Subtotal $(95 \% \mathrm{CI})$

Total events

$\begin{array}{rr}114 & 12 \\ 5 & \\ 38 & \\ 43 & \\ & 23\end{array}$

200

121
8
42
60
231

$101 \quad 108 \quad 39.4 \%$

$94.1 \%$

$1.01[0.94,1.08]$

$1.88[0.64,5.46]$

$33 \quad 42 \quad 32.1 \%$

1. $15[0.96,1.39]$

$1.39[1.04,1.86]$

$1.17[0.93,1.47]$

Heterogeneity. $\mathrm{Tau}^{2}=0.03 ; \mathrm{Chi}^{2}=12.36, \mathrm{df}=3\left(\mathrm{P}=0.006 \mathrm{j} ; \mathrm{I}^{2}=76 \%\right.$

Test for overall effect: $Z=1.32(P=0.19$ )

\subsubsection{HEC}

Tan et al, 2009

Shumway et al, 2015

wiang et al, 2015

Yeo et al, 2020

Subtotal $(95 \% \mathrm{CI})$

$\begin{array}{rr}51 & 56 \\ 5 & 8 \\ 38 & 42 \\ 43 & 60 \\ & 166\end{array}$
$\begin{array}{rrr}41 & 46 & 40.8 \% \\ 3 & 9 & 2.9 \%\end{array}$
$\begin{array}{lll}33 & 42 & 33.8 \%\end{array}$

$1.02[0.90,1.16]$

$1.88[0.64,5.46]$

$1.15[0.96,1.39]$

$1.39[1.04,1.86]$

$1.16[0.96,1.40]$

Total events

137

108

Heterogeneity. Tau $^{2}=0.02 ; \mathrm{Chi}^{2}=6.81, \mathrm{df}=3(\mathrm{P}=0.08) ; \mathrm{I}^{2}=56 \%$

Test for overall effect: $Z=1.56(\mathrm{P}=0.12$ )

\subsubsection{MEC}

Tan et al, 2009

Subtotal $(95 \% \mathrm{CI})$

$63 \quad 65$

Total events

63

65

60

$62 \quad 100.0 \%$

$1.00[0.94,1.07]$

Heterogeneity. Not applicable

Test for overall effect: $Z=0.05(P=0.96)$

\subsubsection{Olanzapine $10 \mathrm{mg}$}

Tan et al, 2009

Shumway et al, 2015

Wang et al, 2015

Yeo et al, 2020

Subtotal $(95 \% \mathrm{CI})$

Total events

$\begin{array}{rr}114 & 12 \\ 5 & \\ 38 & 4 \\ 43 & 6 \\ & 23\end{array}$

200

200

168

60

$62100.0 \%$

$1.00[0.94,1.07]$

Test for overall effect: $Z=1.32(\mathrm{P}=0.19$ )

\subsubsection{Double-Blind Placebo-Controlled Design}

$\begin{array}{lllllll}\text { Yeo et al, } 2020 & 43 & 60 & 31 & 60 & 100.0 \% & 1.39[1.04,1.86] \\ \text { Subtotal }(95 \% \mathrm{Cl}) & & \mathbf{6 0} & & \mathbf{6 0} & \mathbf{1 0 0 . 0 \%} & 1.39[1.04,1.86] \\ \text { Total events } & 43 & & 31 & & & \end{array}$

Heterogeneity. Not applicable

Test for overall effect: $Z=2.20(\mathrm{P}=0.03$ )

3.1.6 Open Controlled Design (Control Arm = All Antiemetics, Except Olanzapine)

$\begin{array}{lrrrrrr}\text { Tan et al, 2009 } & 114 & 121 & 101 & 108 & 68.6 \% & \mathbf{1 . 0 1 [ 0 . 9 4 , 1 . 0 8 ]} \\ \text { Wang et al, 2015 } & 38 & 42 & 33 & 42 & 31.4 \% & \mathbf{1 . 1 5}[0.96,1.39] \\ \text { Subtotal (95\% Cl) } & & \mathbf{1 6 3} & & \mathbf{1 5 0} & \mathbf{1 0 0 . 0 \%} & \mathbf{1 . 0 5}[\mathbf{0 . 9 2}, \mathbf{1 . 2 0}] \\ \text { Total events } & 152 & & 134 & & & \end{array}$

Heterogeneity. Tau $^{2}=0.01 ; \mathrm{Chi}^{2}=2.08, \mathrm{df}=1(\mathrm{P}=0.15) ; \mathrm{I}^{2}=52 \%$

Test for overall effect: $Z=0.73(P=0.46)$

\subsubsection{Open Controlled Design (Control Arm = Antiemetics Not In Olanzapine Arm)}

$\begin{array}{lllllll}\text { Shumway et al, } 2015 & 5 & 8 & 3 & 9 & 100.0 \% & 1.88[0.64,5.46] \\ \text { Subtotal }(95 \% \mathrm{CI}) & & 8 & & 9 & 100.0 \% & 1.88[0.64,5.46] \\ \text { Total events } & 5 & & 3 & & & \end{array}$

Total events

Test for overall effect: $Z=1.15(P=0.25$ )

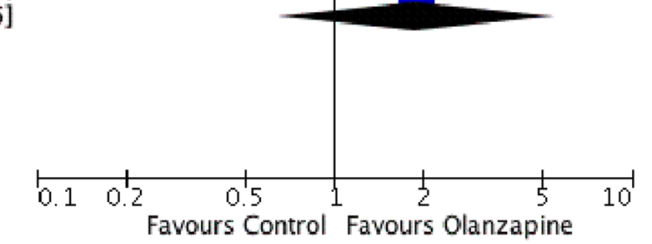

Fig. 3 Efficacy of olanzapine regimens compared to others for the prophylaxis of chemotherapy-induced nausea and vomiting (CINV) — no emesis. 3.1 Acute phase. 3.2 Delayed phase. 3.3 Overall phase 


\section{2}

\begin{tabular}{lrrrrr} 
& \multicolumn{2}{c}{ Olanzapine } & \multicolumn{2}{c}{ Control } \\
Study or Subgroup & Events & Total & Events & Total & Weight \\
\hline 3.2.1 Adults & & & & & \\
Tan et al, 2009 & 112 & 121 & 73 & 108 & $38.4 \%$ \\
Shumway et al, 2015 & 6 & 9 & 5 & 9 & $1.4 \%$ \\
Wang et al, 2015 & 40 & 42 & 34 & 42 & $28.9 \%$ \\
Yeo et al, 2020 & 56 & 60 & 46 & 60 & $31.3 \%$ \\
Subtotal (95\% Cl) & & 232 & & 219 & $100.0 \%$ \\
Total events & 214 & & 158 & &
\end{tabular}

Heterageneity. Tau $^{2}=0.00 ; \mathrm{Chi}^{2}=2.38, \mathrm{df}=3(\mathrm{P}=0.50) ; \mathrm{I}^{2}=0 \%$

Test for overall effect: $Z=5.24$ ( $P<0.00001$ )

\subsubsection{HEC}

Tan et al, 2009

Shumway et al, 2015

Wang et al, 2015

Yeo et al, 2020

Subtotal $(95 \% \mathrm{CI})$

Total events

$\begin{array}{rrrrr}44 & 56 & 26 & 46 & 12.9 \% \\ 6 & 9 & 5 & 9 & 1.9 \% \\ 40 & 42 & 34 & 42 & 40.9 \% \\ 56 & 60 & 46 & 60 & 44.3 \% \\ & 167 & & 157 & 100.0 \%\end{array}$

Risk Ratio

Risk Ratio

Heterogeneity. Tau $^{2}=0.00 ; \mathrm{Chi}^{2}=1.16, d f=3(\mathrm{P}=0.76) ; \mathrm{I}^{2}=0 \%$

Test for overall effect: $\mathrm{Z}=3.79(\mathrm{P}=0.0002)$

\subsubsection{MEC}

$\begin{array}{lcccccr}\text { Tan et al, } 2009 & 58 & 65 & 47 & 62 & 100.0 \% & 1.18[1.00,1.39] \\ \text { Subtotal }(95 \% \mathbf{C l}) & & \mathbf{6 5} & & \mathbf{6 2} & \mathbf{1 0 0 . 0 \%} & \mathbf{1 . 1 8}[1.00, \mathbf{1 . 3 9}] \\ \text { Total events } & 58 & & 47 & & & \end{array}$

Heterogeneity. Not applicable

Test for overall effect: $Z=1.95(\mathrm{P}=0.05)$

\section{2 .4 Olanzapine $10 \mathrm{mg}$}

$\begin{array}{lrrrrrr}\text { Tan et al, 2009 } & 112 & 121 & 73 & 108 & 38.4 \% & 1.37[1.19,1.58] \\ \text { Shumway et al, 2015 } & 6 & 9 & 5 & 9 & 1.4 \% & 1.20[0.57,2.53] \\ \text { Wang et al, 2015 } & 40 & 42 & 34 & 42 & 28.9 \% & 1.18[1.00,1.38] \\ \text { Yeo et al, 2020 } & 56 & 60 & 46 & 60 & 31.3 \% & 1.22[1.04,1.42] \\ \text { Subtotal (95\% Cl) } & & 232 & & 219 & \mathbf{1 0 0 . 0 \%} & 1.26[1.16,1.38] \\ \text { Total events } & 214 & & 158 & & & \end{array}$

Heterageneity. Tau $^{2}=0.00 ;$ Chi $^{2}=2.38, \quad$ df $=3(\mathrm{P}=0.50) ; \mathrm{I}^{2}=0 \%$

Test for overall effect: $Z=5.24(\mathrm{P}<0.00001$ )

\subsubsection{Double-Blind Placebo-Controlled Design

$\begin{array}{lllllll}\text { Yeo et al, } 2020 & 56 & 60 & 46 & 60 & 100.0 \% & 1.22[1.04,1.42] \\ \text { Subtotal }(95 \% \mathrm{Cl}) & & \mathbf{6 0} & & \mathbf{6 0} & \mathbf{1 0 0 . 0 \%} & 1.22[1.04,1.42] \\ \text { Total everts } & 56 & & 46 & & & \end{array}$

Heterogeneity. Not applicable

Test for overall effect: $Z=2.49(\mathrm{P}=0.01)$

$\begin{array}{lrrrrrr}\text { 3.2.6 Open Controlled Design (Control Arm } & \text { All Antiemetics, Except Olanzapine) } \\ \text { Tan et al, } 2009 & 112 & 121 & 73 & 108 & 53.4 \% & 1.37[1.19,1.58] \\ \text { Wang et al, } 2015 & 40 & 42 & 34 & 42 & 46.6 \% & 1.18[1.00,1.38] \\ \text { Subtotal (95\% CI) } & & 163 & & 150 & \mathbf{1 0 0 . 0 \%} & \mathbf{1 . 2 8}[1.09,1.49] \\ \text { Total events } & 152 & & 107 & & & \end{array}$

Heterageneity. Tau $^{2}=0.01 ; \mathrm{Chi}^{2}=2.09, \mathrm{df}=1(\mathrm{P}=0.15) ; \mathrm{I}^{2}=52 \%$

Test for overall effect: $Z=3.10(\mathrm{P}=0.002)$

\subsubsection{Open Controlled Design (Control Arm = Antiemetics Not In Olanzapine Arm)}

$\begin{array}{lllllll}\text { Shumway et al, 2015 } & 6 & 9 & 5 & 9 & 100.0 \% & 1.20[0.57,2.53] \\ \text { Subtotal }(95 \% \mathrm{Cl}) & & 9 & & 9 & 100.0 \% & 1.20[0.57,2.53] \\ \text { Total events } & 6 & & 5 & & & \end{array}$

Total events
Heterogeneity. Not applicable

Test for overall effect: $z=0.48(\mathrm{P}=0.63)$

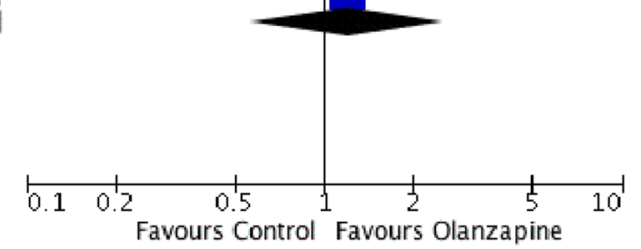

Fig. 3 (continued) 
3.3

Olanzapine Control Risk Ratio

Study or Subgroup

Events Total Events Total Weight $\mathrm{M}-\mathrm{H}$, Random, 95\% Cl

3.3.1 Adults

Tan et al, 2009

Shumway et al, 2015

Jeon et al, 2019

Yeo et al, 2020

Subtotal $(95 \% \mathrm{CI})$

Total events

$\begin{array}{rr}102 & 12 \\ 4 & \\ 20 & 29 \\ 41 & 60 \\ & 219\end{array}$

167

121
9
29
60
219

$\begin{array}{rrr}73 & 108 & 69.7 \% \\ 2 & 9 & 1.1 \% \\ 14 & 25 & 12.1 \% \\ 24 & 60 & 17.0 \% \\ & 202 & 100.0 \%\end{array}$

$1.25[1.07,1.45]$

$2.00[0.48,8.31]$

$1.23[0.81,1.88]$

$1.71[1.20,2.44]$

$1.32[1.13,1.54]$

$$
113
$$

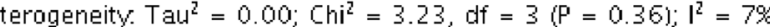

Test for overall effect: $Z=3.59(\mathrm{P}=0.0003)$

\subsubsection{HEC}

Tan et al, 2009

Shumway et al, 2015

Yeo et al, 2020

Subtotal $(95 \% \mathrm{Cl})$

$\begin{array}{rr}44 & 56 \\ 4 & 9 \\ 41 & 60 \\ & 125\end{array}$

$\begin{array}{rrr}26 & 46 & 58.8 \% \\ 2 & 9 & 2.4 \% \\ 24 & 60 & 38.8 \% \\ & 115 & 100.0 \%\end{array}$

$1.39[1.04,1.85]$

$2.00[0.48,8.31]$

$1.71[1.20,2.44]$

$1.52[1.22,1.89]$

$89-52$

Heterogeneity. $\mathrm{Tau}^{2}=0.00 ; \mathrm{Chi}^{2}=0.97, \mathrm{df}=2(\mathrm{P}=0.62) ; \mathrm{I}^{2}=0 \%$

Test for overall effect: $Z=3.71(\mathrm{P}=0.0002)$

\subsubsection{MEC}

Tan et al, 2009

Jeon et al, 2019

Subtotal $(95 \% \mathrm{Cl})$

$\begin{array}{ll}58 & 65 \\ 20 & 29 \\ & 9\end{array}$

65
29
94
$\begin{array}{lll}47 & 62 & 87.0 \%\end{array}$
$25 \quad 13.0 \%$

$78-61$

Heterogeneity. $\mathrm{Tau}^{2}=0.00 ; \mathrm{Chi}^{2}=0.04, \mathrm{df}=1(\mathrm{P}=0.84) ; \mathrm{I}^{2}=0 \%$

Test for overall effect: $z=2.16(\mathrm{P}=0.03$ )

\subsubsection{Olanzapine $10 \mathrm{mg}$}

Tan et al, 2009

Shumway et al, 2015

$102 \quad 121$

49

$20 \quad 29$

Jeon et al, 2019

Yeo et al, 2020

$41 \quad 60$

$$
\begin{array}{r}
60 \\
219
\end{array}
$$

Total events

167

$\begin{array}{rrrr}121 & 73 & 108 & 69.7 \% \\ 9 & 2 & 9 & 1.1 \% \\ 29 & 14 & 25 & 12.1 \% \\ 60 & 24 & 60 & 17.0 \% \\ 219 & & 202 & 100.0 \%\end{array}$

$1.25[1.07,1.45]$

$2.00[0.48,8.31]$

$1.23[0.81,1.88]$

$1.71[1.20,2.44]$

$1.32[1.13,1.54]$

Heterogeneity. $\mathrm{Tau}^{2}=0.00 ; \mathrm{Chi}^{2}=3.23, \mathrm{df}=3(\mathrm{P}=0.36) ; \mathrm{I}^{2}=7 \%$

Test for overall effect: $z=3.59(\mathrm{~F}=0.0003$ )

\subsubsection{Double-Blind Placebo-Controlled Design}

$\begin{array}{lllllrr}\text { Jeon et al, } 2019 & 20 & 29 & 14 & 25 & 43.5 \% & 1.23[0.81,1.88] \\ \text { Yeo et al, 2020 } & 41 & 60 & 24 & 60 & 56.5 \% & 1.71[1.20,2.44] \\ \text { Subtotal }(\mathbf{9 5 \%} \mathbf{C l}) & & \mathbf{8 9} & & \mathbf{8 5} & \mathbf{1 0 0 . 0 \%} & \mathbf{1 . 4 8}[\mathbf{1 . 0 8}, \mathbf{2 . 0 4}]\end{array}$

6138

Heterageneity. $\mathrm{Tau}^{2}=0.01 ; \mathrm{Chi}^{2}=1.37, \mathrm{df}=1(\mathrm{P}=0.24) ; \mathrm{I}^{2}=27 \%$

Test for overall effect: $Z=2.40(\mathrm{P}=0.02)$

$\begin{array}{lcccccc}\text { 3.3.6 Open Controlled Design (Control Arm = All Antiemetics, Except Olanzapine) } \\ \begin{array}{l}\text { Tan et al, } 2009 \\ \text { Subtotal }(95 \% \mathrm{Cl})\end{array} & 102 & 121 & 73 & 108 & 100.0 \% & 1.25[1.07,1.45] \\ \text { Total events } & & 121 & & 108 & \mathbf{1 0 0 . 0 \%} & \mathbf{1 . 2 5}[1.07,1.45] \\ & 102 & & 73 & & & \end{array}$

Heterageneity. Not applicable

Test for overall effect: $Z=2.86(\mathrm{P}=0.004)$

\subsubsection{Open Controlled Design (Control Arm = Antiemetics Not In Olanzapine Arm)}

Shumway et al, 2015

Subtotal $(95 \% \mathrm{CI})$

4

9

$9100.0 \%$

$9100.0 \%$

$2.00[0.48,8.31]$

Total events

42

Heterageneity. Not applicable

Test for overall effect: $z=0.95(\mathrm{P}=0.34)$

Fig. 3 (continued)
Risk Ratio

M-H, Random, 95\% Cl

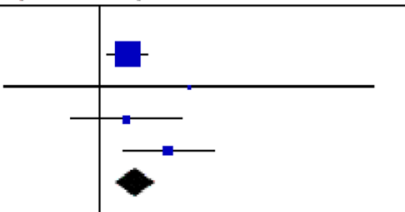




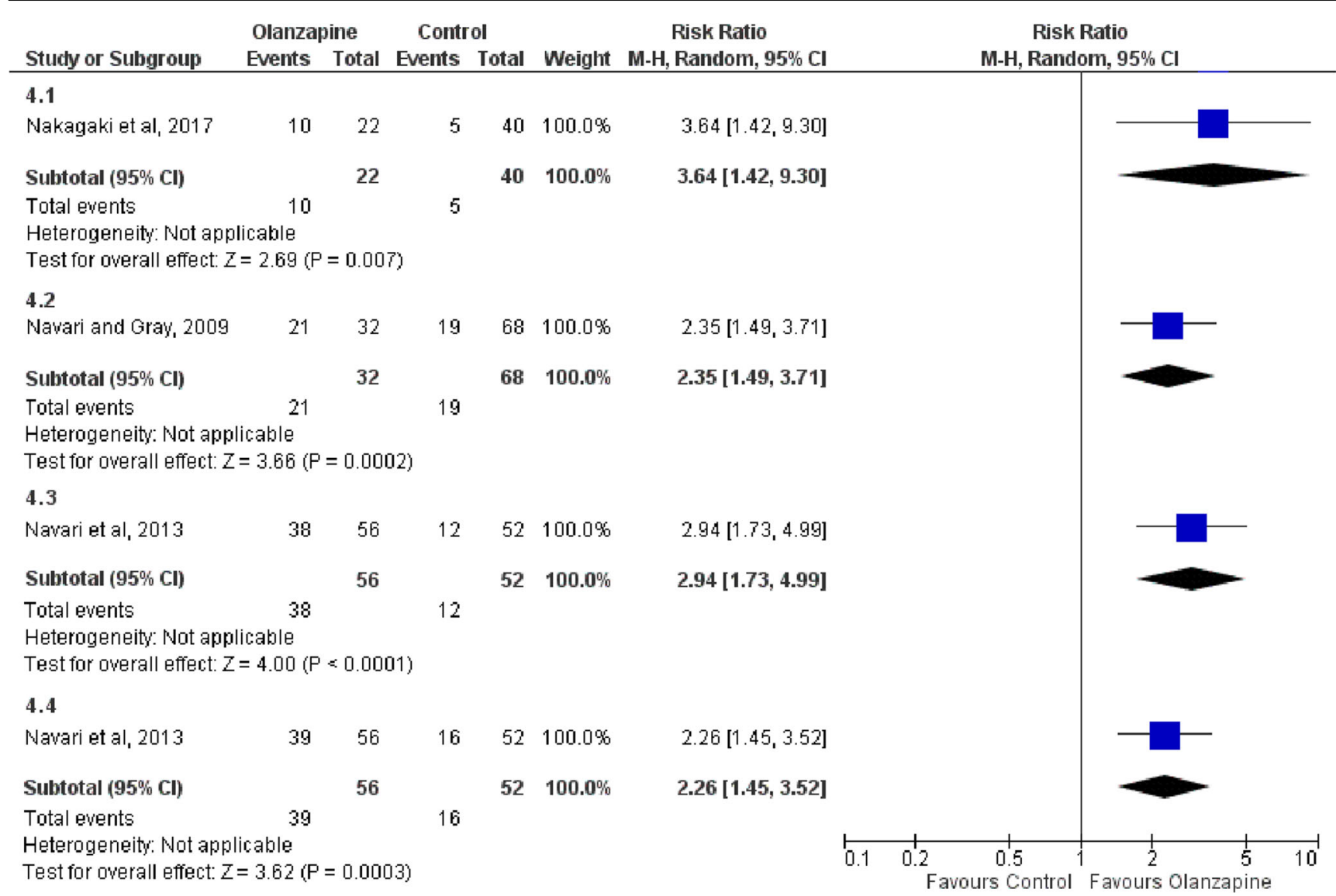

Fig. 4 Efficacy of olanzapine regimens compared to others for the rescue of breakthrough chemotherapy-induced nausea and vomiting (CINV). 4.1 Complete response - acute phase. 4.2 Complete response - overall phase. 4.3 No nausea - overall phase. 4.4 No Emesis - overall phase

For studies reporting on HEC patients, olanzapine is statistically and clinically superior in seven of nine efficacy endpoints in the prophylaxis setting; only complete emetic control in the acute and overall phases were not statistically different from comparative regimens. Meta-analysis results among studies employing 10-mg doses and among studies comparing olanzapine to placebo-controlled regimens indicated olanzapine as statistically and clinically superior in eight of nine efficacy endpoints for prophylaxis of CINV, with the exception of complete emetic control in the acute phase. These results support the international clinical guidelines [28-30] in their recommendation of 10-mg olanzapine in addition to standard antiemetic regimens for the prophylaxis of CINV among HEC patients.

Furthermore, this review includes important subgroup analyses not previously conducted among prophylactic studies, namely meta-analyzing studies reporting on MEC patients and 5-mg olanzapine dosing. Olanzapine is both statistically and clinically superior in only three of six efficacy endpoints where a 5-mg dosage is employed-complete response in the acute, delayed, and overall phases. However, it is important to note that over 800 patients across 4 studies were metaanalyzed for the efficacy endpoints of complete response; there was much less statistical power relative to meta- analyses looking at HEC patients alone. Furthermore, even for the efficacy endpoints of complete response, these metaanalysis results are much more fragile and less certain than those pertaining to olanzapine administered at $10-\mathrm{mg}$ dose studies. Olanzapine may potentially be superior to comparative regimens when administered in 5-mg doses as indicated by point estimates, but the paucity of data results in low statistical power to find these differences statistically significant. Olanzapine has also recently been reported to be effective at 5 $\mathrm{mg}$ doses in controlling nausea and vomiting, unrelated to chemotherapy, for patients with advanced cancer [67]. More RCTs are needed in the CINV setting, to evaluate the efficacy of 5-mg olanzapine doses compared to non-olanzapinecontaining regimens. Studies comparing $5-\mathrm{mg}$ doses to 10 $\mathrm{mg}$ doses are also encouraged; an abstract recently presented by Mukhopadhyay et al. suggests that 5-mg and 10-mg doses may have similar efficacy, although it has no description of drop out patients or chemotherapy regimens in either arm, and no statistical calculations were published [39].

In the MEC setting, olanzapine is reported to be statistically and clinically superior in two of nine efficacy endpoints only - no nausea in the delayed phase, and no emesis in the overall phase. However, as with the results from the metaanalysis of 5-mg doses, there is a paucity of data in this setting. 
The results are less robust compared to those in the HEC setting, with the recent clinical trials having noticeable impacts on the summary effect size. More RCTs in this setting would allow for a better understanding of olanzapine's true efficacy for MEC patients.

Olanzapine is reported to be clinically and statistically superior than other regimens for the rescue of breakthrough CINV. However, this review's results are only supported by one included study for each efficacy endpoint. Results should be interpreted with caution. In both the prophylactic and rescue setting, olanzapine is reported to be equally as safe as other regimens. However, this too should be interpreted with caution, as the key adverse event of sedation is not routinely reported - many studies commonly reported only on serious (i.e., grade 3 or greater toxicity) adverse events, an observation also noted by our group several years ago [26]. It has been well-documented that olanzapine is a strong sedative, and patients commonly experience fatigue, drowsiness, and reduced general activity [20]. In the interest of reducing adverse events, further exploring the reduction of the dosage of olanzapine (i.e. more RCTs reporting on 5-mg olanzapine doses) is encouraged.

This review was not without limitations. Ideally, the protocol would have been registered on PROSPERO; given the COVID 19 pandemic, this was not a feasible optionprotocol registration would have required several months, while in hindsight our review was already completed. There were numerous instances where there were high levels of heterogeneity; a random-effects model was applied in all circumstances to try to appropriately account for this. As well, as is the nature of meta-analyses, the results suffer from any intrinsic biases from included RCTs; over half of the studies have notable concerns of bias due to lack of blinding.

In conclusion, olanzapine is effective and safe for the prophylaxis and rescue of CINV. It has been well-documented in the HEC setting and when administered at 10-mg doses; it is statistically and clinically superior to comparative regimens, but its sedative properties can make it difficult to use in outpatient settings. It is unclear if olanzapine is effective in the MEC setting and when administered at a lower 5-mg dose, and further RCTs are needed for a more definitive conclusion. The sedative effect associated with $10 \mathrm{mg}$ of olanzapine further corroborates the need for more investigations into using olanzapine at lower doses.

Supplementary Information The online version contains supplementary material available at https://doi.org/10.1007/s00520-020-05935-7.

Authors' contributions All authors contributed substantially to conception and design, or acquisition of data, or analysis and interpretation of data. All authors contributed in drafting the article or revising it critically for important intellectual content. All authors approved the version to be published.
Data availability N/A

\section{Compliance with ethical standards}

Conflict of interest Dr Lock reports consulting fees from Ferring, Abbvie, Sanofi, and AstraZeneca in the past 10 years outside the submitted work. Dr Herrstedt reports personal fees from SOBI and GSK outside the submitted work. Dr Aapro reports personal fees and non-financial support from the Multinational Association for Supportive Care in Cancer, personal fees and non-financial support from European Society of Medical Oncology, personal fees and non-financial support from the European Cancer Organisation, grants and personal fees from Helsinn, personal fees from Tesaro, grants and personal fees from Sandoz, personal fees from Merck USA, personal fees from Vifor, personal fees from Pfizer, personal fees from Taiho, and personal fees from Kyowa Kirin, outside the submitted work. The other authors declare that they have no conflict of interest.

\section{Ethics approval N/A}

Consent to participate N/A

Consent to publication N/A

Code availability N/A

\section{References}

1. Cohen L, de Moor CA, Eisenberg P et al (2007)Chemotherapyinduced nausea and vomiting: incidence and impact on patient quality of life at community oncology settings. Support Care Cancer 15: 491-503

2. Pollera CF, Giannarelli D (1989) Prognostic factors influencing cisplatin-induced emesis: definition and validation of a predictive logistic model. Cancer 64:1117-1122

3. du Bois A, Meerpohl HG, Vach W et al (1992) Course, patterns and risk-factors for chemotherapy-induced emesis in cisplatinpretreated patients: a study with ondansetron. Eur J Cancer 28: $450-457$

4. Osoba D, Zee B, Pater J, Warr D, Latreille J, Kaizer L (1997) Determinants of postchemotherapy nausea and vomiting in patients with cancer. J Clin Oncol 15:116-123

5. Molassiotis A, Aapro M, Dicato M, Gascon P, Novoa SA, Isambert N, Burke TA, Gu A, Roila F (2014) Evaluation of risk factors predicting chemotherapy-related nausea and vomiting: results from a European prospective observational study. J Pain Symptom Manag 47:839-848

6. Chow R, Warr DG, Navari RM, Tsao M, Milakovic M, Popovic M, Chiu L, Lam H, DeAngelis C (2018) Efficacy and safety of 1-day versus 3-day dexamethasone for the prophylaxis of chemotherapyinduced nausea and vomiting: a systematic review and metaanalysis of randomized controlled trials. J Hosp Manag Health Policy 2:25

7. Chow R, Valdez C, Chow N, Zhang D, Im J, Sodhi E, Lock M (2020) Oral cannabinoid for the prophylaxis of chemotherapyinduced nausea and vomiting - a systematic review and meta-analysis. Support Care Cancer 28:2095-2103

8. Gralla RJ, Itri LM, Pisko SE, Squillante AE, Kelsen DP, Braun DW Jr, Bordin LA, Braun TJ, Young CW (1981) Antiemetic efficacy of high-dose metoclopramide: randomized trials with placebo and prochlorperazine in patients with chemotherapy-induced nausea and vomiting. N Engl J Med 305:905-909 
9. Chow R, Tsao M, Chiu L, Popovic M, Milakovic M, Lam H, DeAngelis C (2018) Efficacy of the combination of neurokinin-1 receptor antagonist, palonosetron, and dexamethasone compared to others for the prophylaxis of chemotherapy-induced nauseas and vomiting: a systematic review and meta-analysis of randomized controlled trials. Ann Palliat Med 7:221-223

10. Jordan K, Blattermann L, Hinke A et al (2018) Is the addition of a neurokinin-1 receptor antagonist beneficial in moderately emetogenic chemotherapy? - A systematic review and meta-analysis. Support Care Cancer 26:21-32

11. Chow R, Warr DG, Navari RM, Tsao M, Popovic M, Chiu L, Milakovic M, Lam H, DeAngelis C (2018) Should palonosetron be a preferred $5-\mathrm{HT}_{3}$ receptor antagonist for chemotherapy-induced nausea and vomiting? An updated systematic review and metaanalysis. Support Care Cancer 26:2519-2549

12. Brymaster FP, Calligaro D, Falcone J et al (1996)Radio-receptor binding profile of the atypical antipsychotic olanzapine. Neuropsychopharmacology 14:87-96

13. Srivastava M, Brito-Dellan N, Davis MP, Leach M, Lagman R (2003) Olanzapine as an antiemetic in refractory nausea and vomiting in advanced cancer. J Pain Symptom Manag 25:578-582

14. Abe M, Hirashima Y, Kasamatsu Y, Kado N, Komeda S, Kuji S, Tanaka A, Takahashi N, Takekuma M, Hihara H, Ichikawa Y, Itonaga Y, Hirakawa T, Nasu K, Miyagi K, Murakami J, Ito K (2016) Efficacy and safety of olanzapine combined with aprepitant, palonosetron and dexamethasone for preventing nausea and vomiting by cisplatin-based chemotherapy in gynecological cancer: KCOG-G1303 phase II trial. Support Care Cancer 24:675-682

15. Park S, Choi C, Kwon M et al (2015) A phase II trial of palonosetron and olanzapine without dexamethasone for the prevention of chemotherapy-induced nausea and vomiting. Support Care Cancer 23(Suppl 1):S147

16. Navari RM, Einhorn L, Passik S et al (2005) A phase II trial of olanzapine for the prevention of chemotherapy-induced nausea and vomiting: a Hoosier Oncology Group study. Support Care Cancer 13:529-534

17. Navari RM, Einhorn L, Loehrer P et al (2007) A phase II trial of olanzapine for the prevention of chemotherapy-induced nausea and vomiting: a Hoosier Oncology Group study. Support Care Cancer 15:1285-1291

18. Passik S, Navari RM, Jong S et al (2004) A phase I trial of olanzapine (zyprexa) for the prevention of delayed emesis in cancer patients: a Hoosier Oncology Group Study. Cancer Investig 22: 383-388

19. Passik S, Lundberg J, Kirsh K et al (2002) A pilot exploration of the antiemetic activity of olanzapine for the relief of nausea in patients with advanced cancer and pain. J Pain Symptom Manag 23:526-532

20. Chow R, Chiu L, Navari R, Passik S, Chiu N, Popovic M, Lam H, Pasetka M, Chow E, DeAngelis C (2016) Efficacy and safety of olanzapine for the prophylaxis of chemotherapy-induced nausea and vomiting (CINV) as reported in phase I and II studies: a systematic review. Support Care Cancer 24:1001-1008

21. Zhou JG, Huang L, Jin SH et al (2020) Olanzapine combined with 5-hydroxytryptamine type 3 receptor antagonist (5-HT3 RA) plus dexamethasone for prevention and treatment of chemotherapyinduced nausea and vomiting in high and moderate emetogenic chemotherapy: a systematic review and meta-analysis of randomized controlled trials. ESMO Open 6:e000621

22. Bahbah EI, Abdalla AR, Abdelshafy K, Almohandes AD, Menshawy A, Elalem Aziz MA, Ebada MA, Hegab A, Negida A (2019) Should olanzapine be advocated over conventional antiemetics for the prevention of chemotherapy-induced nausea and vomiting? An updated meta-analysis of randomized control trials. Curr Enzym Inhib 15:80-90

23. Sutherland A, Naessens K, Plugge E, Ware L, Head K, Burton MJ, Wee B, Cochrane Pain, Palliative and Supportive Care Group
(2018) Olanzapine for the prevention and treatment of cancerrelated nausea and vomiting in adults. Cochrane Database Syst Rev. https://doi.org/10.1002/14651858.CD012555.pub2

24. Chelkeba L, Gidey K, Mamo A, Yohannes B, Matso T, Melaku T (2017) Olanzapine for chemotherapy-induced nausea and vomiting: systematic review and meta-analysis. Pharm Pract 15:877

25. Yoodee J, Permsuwan U, Nimworapan M (2017) Efficacy and safety of olanzapine for the prevention of chemotherapy-induced nausea and vomiting: a systematic review and meta-analysis. Crit Rev Oncol Hematol 112:113-125

26. Chiu L, Chow R, Popovic M, Navari RM, Shumway NM, Chiu N, Lam H, Milakovic M, Pasetka M, Vuong S, Chow E, DeAngelis C (2016) Efficacy of olanzapine for the prophylaxis and rescue of chemotherapy-induced nausea and vomiting (CINV): a systematic review and meta-analysis. Support Care Cancer 24:2381-2392

27. Hocking CM, Kichenadasse G (2014) Olanzapine for chemotherapy-induced nausea and vomiting: a systematic review. Support Care Cancer 22:1142-1151

28. Hesketh PJ, Kris MG, Basch E et al (2017) Antiemetics: American Society of Clinical Oncology Clinical Practice Guideline Update. J Clin Oncol 28:3240-3261

29. Berger MJ, Ettinger DS, Aston J, Barbour S, Bergsbaken J, Bierman PJ, Brandt D, Dolan DE, Ellis G, Kim EJ, Kirkegaard S, Kloth DD, Lagman R, Lim D, Loprinzi C, Ma CX, Maurer V, Michaud LB, Nabell LM, Noonan K, Roeland E, Rugo HS, Schwartzberg LS, Scullion B, Timoney J, Todaro B, Urba SG, Shead DA, Hughes M (2017) NCCN guidelines insights: antiemesis, versions 2.2017. J Natl Compr Cancer Netw 15:883-893

30. Herrstedt J (2018) The latest consensus on antiemetics. Curr Opin Oncol 30:233-239

31. Shea BJ, Wells G, Hamel C et al (2017) AMSTAR 2: a critical appraisal tool for systematic reviews that include randomised or non-randomised studies of healthcare interventions, or both. BMJ 358:j4008

32. Liberati A, Altman DG, Tetzlaff J, Mulrow C, Gotzsche PC, Ioannidis JPA, Clarke M, Devereaux PJ, Kleijnen J, Moher D (2009) The PRISMA statement for reporting systematic reviews and meta-analyses of studies that evaluate healthcare interventions: explanation and elaboration. BMJ 339:b2700

33. Jordan K, Chan A, Gralla RJ, Jahn F, Rapoport B, Warr D, Hesketh PJ (2017) 2016 updated MASCC/ESMO consensus recommendations: emetic risk classification and evaluation of the emetogenicity of antineoplastic agents. Support Care Cancer 25:271-275

34. Roila F, Molassiotis A, Herrstedt J et al (2016) 2016 MASCC and ESMO guideline update for the prevention of chemotherapy- and radiotherapy-induced nausea and vomiting and of nausea and vomiting in advanced cancer patients. Ann Oncol 26:v119-v133

35. Walsh M, Srinathan SK, McAuley DF et al (2014) The statistical significance of randomized controlled trial results is frequently fragile: a case for a Fragility Index. J Clin Epidemiol 67:622-628

36. Babu G, Saldanha SG, Chinnaglriyappa LK et al (2016) The efficacy, safety and cost benefit of olanzapine versus aprepitant in highly emetogenic chemotherapy: a pilot study from South India. Chemother Res Pract 2016:1-5. https://doi.org/10.1144/2016/3439707

37. Flank J, Schechter T, Gibson P, Johnston DL, Orsey AD, Portwine C, Sung L, Dupuis LL (2018) Olanzapine for prevention of chemotherapy-induced nausea and vomiting in children and adolescents: a multi-center, feasibility study. Support Care Cancer 26: 549-555

38. Osman AAM, Elhassan MMA, AbdElrahim BHA et al (2018) Olanzapine for the prevention of chemotherapy-induced nausea and vomiting: a comparative study from Sudan. J Glob Oncol 4: 1-9. https://doi.org/10.1200/JGO.17.00216

39. Navari RM, Brenner MC (2010) Treatment of cancer-related anorexia with olanzapine and megestrol acetate: a randomized trial. Support Care Cancer 18:951-956 
40. Minatogawa H, Izawa N, Kawaguchi T et al (2019) Phase III study of comparing dexamethasone on day 1 with day 1-4 with combined neurokinin-1 receptor antagonist, palonosetron and olanzapine in cisplatin-based chemotherapy. Support Care Cancer 27(Suppl 1): S38-S39

41. Mukhopadhyay S, Dutta P, Bhattacharya B et al (2018) Low dose olanzapine in chemotherapy induced nausea and vomiting: The ideal 8 pm antiemetic? Support Care Cancer 26(Suppl 1):S79

42. Navari RM, Gray SE (2009) Treatment of chemotherapy-induced breakthrough nausea and vomiting. J Clin Oncol 27:e20536. https:// doi.org/10.1200/jco.2009.27.15_suppl.e20536

43. Navari RM, Nagy CK, Gray SE (2013) The use of olanzapine versus metoclopramide for the treatment of breakthrough chemotherapy-induced nausea and vomiting in patients receiving highly emetogenic chemotherapy. Support Care Cancer 21:16551663

44. Nakagaki M, Barras M, Curley C, Butler JP, Kennedy GA (2017) A randomized trial of olanzapine versus palonosetron versus infused ondansetron for the treatment of breakthrough chemotherapyinduced nausea and vomiting in patients undergoing hematopoietic stem cell transplantation. Support Care Cancer 25:607-613

45. Tan L, Liu J, Liu X, Chen J, Yan Z, Yang H, Zhang D (2009) Clinical research of olanzapine for prevention of chemotherapyinduced nausea and vomiting. J Exp Clin Cancer Res 28:131

46. Navari RM, Gray SE, Kerr AC (2011) Olanzapine versus aprepitant for the prevention of chemotherapy-induced nausea and vomiting: a randomized phase III trial. J Support Oncol 9:188-195

47. Mizukami N, Yamauchi M, Koike K, Watanabe A, Ichihara K, Masumori N, Yamakage M (2014) Olanzapine for the prevention of chemotherapy-induced nausea and vomiting in patients receiving highly or moderately emetogenic chemotherapy: a randomized, double-blind, placebo-controlled study. J Pain Symptom Manag 47:542-550

48. Long C, Knoderer H, Mueller E (2015) A pilot study comparing the addition of olanzapine or aprepitant in an antiemetic regimen for highly emetogenic chemotherapy. Pediatr Blood Cancer 62(Suppl 2):S104

49. Shumway NM, Terrazzino SE, Jones CG (2015) A randomized pilot study comparing olanzapine (Zyprexa) to aprepitant (Emend) for treatment of chemotherapy-induced nausea and vomiting. J Pain Manag 8:233-241

50. Wang X, Wang L, Wang H, Zhang H (2015) Effectiveness of olanzapine combined with ondansetron in prevention of chemotherapy-induced nausea and vomiting of non-small cell lung cancer. Cell Biochem Biophys 72:471-473

51. Navari RM, Nagy CK, Le-Rademacher JL et al (2016) Olanzapine versus fosaprepitant for the prevention of concurrent chemotherapy radiotherapy-induced nausea and vomiting. J Community Support Oncol 14:141-147

52. Navari RM, Qin R, Ruddy KJ, Liu H, Powell SF, Bajaj M, Dietrich L, Biggs D, Lafky JM, Loprinzi CL (2016) Olanzapine for the prevention of chemotherapy-induced nausea and vomiting. $\mathrm{N}$ Engl J Med 375:134-142

53. Mukesh S, Sathya M, Akshay JK (2017) Olanzapine versus aprepitant in the prevention of chemotherapy induced nausea and vomiting (CINV) in breast cancer patients. J Clin Oncol 35:e21670. https://doi.org/10.1200/JCO.2017.35.15 suppl.e21670

54. Mukhopadhyay S, Gagandeep K, Alice KP et al (2017) Role of olanzapine in chemotherapy-induced nausea and vomiting on platinum-based chemotherapy patients: a randomized controlled study. Support Care Cancer 25:145-154

55. Sapkota S, Mahaseth R, Jha K (2017) The use of olanzapine compared to aprepitant as antiemetic for prevention of chemotherapy induced nausea and vomiting in highly emetogenic chemotherapy a randomized trial. Eur J Cancer 72:S168. https://doi.org/10.1016/ S0959-8049(17)30623-8
56. Tran T, Nguyen N, Pham V et al (2017) Olanzapine and omeprazole combination is simple, safe and effective for delayed nausea and vomiting control in adjuvant chemotherapy for early stage breast cancer. Support Care Cancer 25:S72

57. Celio L, Salbene G, Lepori S et al (2019)Short-course olanzapine to prevent delayed emesis following carboplatin/paclitaxel for gynecologic cancer: a randomised study. Tumori J 105:253-258

58. Dulal S, Paudel BD, Neupane P, Shah A, Acharya B, Poudyal BS, Shilpakar R, Wood LA (2019) Randomized phase II trial to compare the efficacy of haloperidol and olanzapine in the control of chemotherapy-induced nausea and vomiting in Nepal. J Glob Oncol 5:1-6. https://doi.org/10.1200/JGO.18.00245

59. Jeon SY, Han HS, Bae WK, Park MR, Shim H, Lee SC, Go SI, Yun HJ, Im YJ, Song EK (2019) A randomized double-blind, placebocontrolled study of the safety and efficacy of olanzapine for the prevention of chemotherapy-induced nausea and vomiting in patients receiving moderately emetogenic chemotherapy: results of the Korean South West Oncology Group (KSWOG) study. Cancer Res Treat 51:90-97

60. Rumyantsev A, Glazkova E, Nasyrova R, Ignatova E, Chitia L, Popova A, Chekini D, Kochetkova Y, Kit S, Elsnukaeva K, Menshikova S, Sekhina O, Pokataev I, Tyulyandina A, Stenina M, Frolova MA, Bulanov A, Fedyanin M, Tryakin A, Tjulandin S (2019) Olanzapine (OLN) versus aprepitant (APR) in patients receiving high-emetogenic chemotherapy: final results of randomized phase II trial. J Clin Oncol 37:11504. https://doi.org/10.1200/ JCO.2019.37.15_suppl.11504

61. Saldanha SC, Dasappa L, Jacob LA, Babu SM, Lokesh KN, Rudresha AH, Lakkavalli RK, Kumar J (2019) Efficacy of olanzapine combination in prevention of nausea \& vomiting in highly emetogenic chemotherapy. Ann Oncol 30:v722

62. Tienchaiananda P, Nipondhkit W, Maneenil K, Sa-nguansai S, Payapwattanawong S, Laohavinij S, Maneechavakajorn J (2019) A randomized, double-blind, placebo-controlled study evaluating the efficacy of combination olanzapine, ondansetron and dexamethasone for prevention of chemotherapy-induced nausea and vomiting in patients receiving doxorubicin plus cyclophosphamide. Ann Palliat Med 8:372-380

63. Hashimoto H, Abe M, Tokuyama O, Mizutani H, Uchitomi Y, Yamaguchi T, Hoshina Y, Sakata Y, Takahashi TY, Nakashima K, Nakao M, Takei D, Zenda S, Mizukami K, Iwasa S, Sakurai M, Yamamoto N, Ohe Y (2020) Olanzapine $5 \mathrm{mg}$ plus standard antiemetic therapy for the prevention of chemotherapy-induced nausea and vomiting (J-FORCE): a multicentre, randomised, double-blind, placebo-controlled, phase 3 trial. Lancet Oncol 21:242249

64. Ithimakin S, Theeratrakul P, Laocharoenkiat A, Nimmannit A, Akewanlop C, Soparattanapaisarn N, Techawattanawanna S, Korphaisarn K, Danchaivijitr P (2020) Randomized, double-blind, placebo-controlled study of aprepitant versus two dosages of olanzapine with ondansetron plus dexamethasone for prevention of chemotherapy-induced nausea and vomiting in patients receiving high-emetogenic chemotherapy. Support Care Cancer 28:53355342. https://doi.org/10.1007/s00520-020-05380-6

65. Vimolchalao V, Sakdejayont S, Wongchanapai P, Sukprakun S, Angspatt P, Thawinwisan W, Chenaksara P, Sriuranpong V, Vinayanuwatikun C, Parinyanitikun N, Poovorawan N, Tanasanvimon S (2020) The efficacy and safety of the addition of olanzapine to ondansetron and dexamethasone for prevention of chemotherapy-induced nausea and vomiting in patients receiving highly emetogenic chemotherapy. Int J Clin Oncol 25:396-402

66. Yeo W, Lau TKH, Li L, Lai KT, Pang E, Cheung M, Chan VTC, Wong A, Soo WMT, Yeung VTY, Tse T, Lam DCM, Yeung EWM, Ng KPK, Tang NLS, Tong M, Suen JJS, Mo FKF (2020) A randomized study of olanzapine-containing versus standard 
antiemetic regimens for the prevention of chemotherapy-induced nausea and vomiting in Chinese breast cancer patients. Breast 50: 30-38

67. Navari RM, Pywell CM, Le-Rademacher JG et al (2020) Olanzapine for the treatment of advanced cancer-related chronic nausea and/or vomiting. JAMA Oncol 6:895-899. https://doi.org/ 10.1001/jamaoncol.2020.1052

Publisher's note Springer Nature remains neutral with regard to jurisdictional claims in published maps and institutional affiliations. 